\title{
A switch from canonical to noncanonical Wnt signaling mediates early differentiation of human neural stem cells.
}

Nora Bengoa-Vergniory ${ }^{1}$, Irantzu Gorroño-Etxebarria ${ }^{1}$, Itxaso González Salazar ${ }^{1}$ and Robert M. Kypta ${ }^{1,2}$

${ }^{1}$ Cell Biology and Stem Cells Unit, CIC bioGUNE, Spain and ${ }^{2}$ Department of Surgery and Cancer, Imperial College London, UK

Author contributions: Nora Bengoa-Vergniory: conception and design, collection and assembly of data, data analysis and interpretation, manuscript writing, final approval of manuscript; Irantzu Gorroño-Etxebarria: administrative and technical support; Itxaso González Salazar: collection of data and technical support; Robert Kypta: conception and design, financial support, data analysis and interpretation, manuscript writing, final approval of manuscript.

Corresponding Author: Robert Kypta, Cell Biology and Stem Cells Unit, CIC bioGUNE, Parque Tecnológico de Vizcaya, Derio 48160, Spain, Tel.: +34 94406 1321;

rkypta@,cicbiogune.es

Funding: Basque Department of Education BFI-2010-129, Spanish Ministry of Science and Innovation (SAF2011-30494) and the Department of Industry, Tourism and Trade (Etortek) and Department of Innovation Technology of the Government of the Autonomous Community of the Basque Country.

Keywords: Neural Stem Cell, Neural differentiation, Signal transduction, Cell signaling 


\begin{abstract}
$\mathrm{Wnt} / \beta$-catenin signaling is essential for neurogenesis but less is known about $\beta$-cateninindependent Wnt signals. We show here that Wnt/AP-1 signaling drives differentiation of human embryonic stem (hES) cell and induced pluripotent stem (iPS) cell-derived neural progenitor cells. Neuronal differentiation was accompanied by a reduction in $\beta$-catenin/Tcfdependent transcription and target gene expression, increased levels and/or phosphorylation of ATF2, CREB and c-Jun and increased AP-1-dependent transcription. Inhibition of Wnt secretion using the porcupine inhibitors IWP-2 and Wnt-C59 blocked neuronal differentiation, while activation or inhibition of $\mathrm{Wnt} / \beta$-catenin signaling had no effect. Neuronal differentiation increased expression of several Wnt genes, including WNT3A, silencing of which reduced differentiation. Addition of recombinant Wnt-3a to cells treated with IWP-2 or Wnt-C59 increased AP-1 levels and restored neuronal differentiation. The effects of Wnt-3a could not be blocked by addition of Dkk-1 or IWR-1, suggesting the involvement of noncanonical signaling. Consistent with this, restoration of neuronal differentiation by Wnt-3a was reduced by inhibition of Jun N-terminal kinase (JNK) and by gene silencing of ATF2. Together, these observations suggest that $\beta$-catenin-independent Wnt signals promote NSC differentiation in a signaling pathway involving Wnt-3a, JNK and
\end{abstract} ATF2. 


\section{INTRODUCTION}

Wnt proteins play roles in many cellular and physiological processes, regulating cell proliferation, differentiation, migration and patterning during development and tissue homeostasis in adults, primarily through their effects on stem/progenitor cells [1,2]. In the canonical Wnt pathway, $\beta$-catenin is degraded by a protein complex containing Axin, glycogen synthase kinase-3 (GSK-3), casein kinase 1 (CK1) and adenomatous polyposis coli (APC). When a Wnt protein binds to frizzled (FZD) and low-density lipoprotein receptorrelated protein (LRP5/6) receptors, Axin and disheveled (DVL) are recruited to the membrane, leading to disruption of the degradation complex, $\beta$-catenin stabilization and entry into the nucleus and activation of T-cell factor/lymphoid enhancer factor-1 (TCF/LEF) target genes [1]. In addition, noncanonical Wnt signals affect DVL and other intracellular components to activate the Planar Cell Polarity (PCP) and Wnt-Calcium pathways [3]. In the PCP pathway, FZD receptors activate a cascade that involves the small GTPases and c-Jun $\mathrm{N}$-terminal kinase (JNK), leading to changes in the cytoskeleton and activation of Activator Protein-1 (AP-1) family transcription factors, including activating transcription factor 2 (ATF2), c-Jun and cyclic AMP response element-binding protein (CREB) [4]. Noncanonical Wnt ligands interact with numerous receptors, including receptor tyrosine kinase-like orphan receptor (ROR1/2), receptor-like tyrosine kinase (RYK) and van gogh-like (VANGL1/2) [5]. Adding to this complexity, the Wnt response can differ depending on the cellular or Wnt receptor context $[6,7]$, further underlining the importance of knowing the 'Wnt status' of cells at different stages of differentiation.

Many studies highlight the critical role of Wnt signaling in neural differentiation, but the details remain unclear, with reports of increased and decreased Wnt/ $\beta$-catenin signaling, as well as changes in noncanonical Wnt signaling, being required $[8,9]$. The importance of 
$\mathrm{Wnt} / \beta$-catenin signaling may lie in its role in maintaining neural stem/progenitor cell (NSC) proliferation: inhibition of $\mathrm{Wnt} / \beta$-catenin signaling reduces NSC proliferation in neurospheres [10] and loss of the secreted Wnt antagonist Dickkopf-1 (Dkk-1) increases the number of hippocampal neural progenitors [11]. Constitutive activation of $\mathrm{Wnt} / \beta$-catenin signaling, however, disrupts NSC proliferation, migration and differentiation [12, 13]. Moreover, basal Wnt/ $\beta$-catenin signaling has been shown to repress neuronal differentiation of human NSCs [14], and its inhibition promotes neural precursor specification [15]. Thus, tight control of $\mathrm{Wnt} / \beta$-catenin signaling is important for proper differentiation. Comparatively little is known about noncanonical Wnt signaling in this context [16]. Wnt-5a has been shown to cooperate with Wnt-1 to regulate morphogenesis and neurogenesis during the generation of mouse midbrain dopaminergic (DA) neurons [17], and Wnt-4 and Wnt-11 play distinct roles in neuronal differentiation of NT2 cells [9]. The downstream effectors of these Wnts are not known, but candidates include ATF2 and CREB, which are implicated in neurogenesis [18, 19].

The generation of new neurons from NSCs takes place in the subventricular zone and in the subgranular zone of the hippocampus [20]. Wnt signaling promotes hippocampal neurogenesis by stimulating neuroblast proliferation and instructing neuronal fate [21] (reviewed in [16]). Since aberrant neurogenesis is believed to be involved in epilepsy, stroke and neurodegenerative disease [22], a better understanding of the roles of Wnt signaling in NSCs can lead to new treatments for these disorders. Here we have examined Wnt signaling in human embryonic stem cell-derived neural progenitor cells, which resemble primary NSCs and have been used to study human NSC differentiation [23-25], and also in human induced pluripotent stem (iPS) cell-derived neural progenitors, which have potential uses in cell transplantation therapy, modeling of human disease and drug development [26]. Our results indicate that a switch from Wnt//-catenin to Wnt/AP-1 signaling takes place during NSC 
differentiation. We further find that inhibition of Wnt secretion blocks neuronal differentiation, and that its restoration by Wnt-3a involves activation of JNK/ATF2. Our observations are consistent with a model in which noncanonical Wnt signaling, rather than Wnt/ $\beta$-catenin signaling, promotes human NSC differentiation.

\section{MATERIALS AND METHODS}

\section{Cell culture and drug treatments}

Human neural progenitor (hNP) cells (Aruna Biomedical, Athens, GA) were grown at $37^{\circ} \mathrm{C}$, 5\% $\mathrm{CO}_{2}$ in Neurobasal Medium with $1 \times$ B27 Supplement, $1 \times$ Penicillin/Streptomycin and 2 mM L-Glutamine (Gibco) plus 20 ng/ml basic Fibroblast Growth Factor (bFGF) (Life Technologies) and $10 \mathrm{ng} / \mathrm{ml}$ Leukemia Inhibitory Factor (ProSpec) on Matrigel-coated plates. To coat plates, Matrigel (BD) was thawed on ice and diluted in cold Neurobasal Medium 1:200 $(8.95 \mathrm{mg} / \mathrm{ml})$, applied to pre-cooled plates and left overnight at $4^{\circ} \mathrm{C}$. Plates were then washed with PBS, left in a laminar flow hood to dry for $15-20 \mathrm{~min}$ and then stored at $4^{\circ} \mathrm{C}$ for up to two weeks. Before use, plates were rehydrated with PBS for 30 min at $37^{\circ} \mathrm{C}, 5 \% \mathrm{CO}_{2}$. Cells were fed every other day and the day after plating and were passaged at 1:2 or 1:3 and used for up to 20 passages. Differentiation was induced by removal of bFGF from the medium. Unless stated otherwise, cells were differentiated for 3 days. Human iPS cell-derived neural progenitor cells (EMD Millipore, Billerica, MA) were grown on Matrigel-coated plates in ENStem-A ${ }^{\mathrm{TM}}$ Neural Expansion Medium (Millipore) with Penicillin/Streptomycin and LGlutamine (as above) and $40 \mathrm{ng} / \mathrm{ml} \mathrm{bFGF.} \mathrm{Cells} \mathrm{were} \mathrm{fed} \mathrm{every} \mathrm{other} \mathrm{day} \mathrm{and} \mathrm{the} \mathrm{day} \mathrm{after}$ plating and were passaged at 1:2 or 1:3 and used for up to 5 passages. Differentiation was induced by culture in Neurobasal Medium with 1 x B27 Supplement, Penicillin/Streptomycin, $2 \mathrm{mM}$ L-Glutamine and $200 \mathrm{uM}$ Ascorbic Acid (Sigma), as above. Unless stated otherwise, cells were treated every day for 5 days. IWP-2 (Calbiochem) was used at $2 \mathrm{uM}$, diluted in 
medium and filtered before use. Equal amounts of DMSO (Sigma) were diluted in medium and filtered before use as controls. CHIR99021 (Calbiochem), iCRT14 (Tocris), IWR-1-endo (Calbiochem) and JNK Inhibitor VIII (Millipore) and corresponding volumes of DMSO as a control, were diluted in medium prior to use at $500 \mathrm{nM}, 1 \mathrm{uM}, 10 \mathrm{uM}$ and $10 \mathrm{uM}$, respectively. Wnt-C59 (Cellagen Technology) was resuspended in DMSO, diluted in medium, and used at $100 \mathrm{nM}$ or as indicated in each experiment. Rho kinase inhibitor Y27632 (Abcam) and CAMKII inhibitor myr-AIP (Millipore) were solubilized in water and used as indicated. Recombinant Wnt-7a, Wnt-5a, Wnt-3a and Dkk-1 (R\&D Systems) were used at $400 \mathrm{ng} / \mathrm{ml}, 200 \mathrm{ng} / \mathrm{ml}, 100 / 200 \mathrm{ng} / \mathrm{ml}$ and $100 \mathrm{ng} / \mathrm{ml}$, respectively, with corresponding dilutions of vehicle (1\% CHAPS) as a control. If not stated otherwise, cells were treated every day for 3 days; for longer treatments cells were treated every other day.

\section{RNA analysis}

RNA was extracted using the PureLink RNA Micro Kit (Invitrogen), according to manufacturer's instructions. RNA purity and quantity were measured on a Nanodrop spectrophotometer and 1 ug of total RNA was transcribed with M-MLV-RT (Invitrogen) in the presence of RNaseOUT (Invitrogen), according to manufacturer's instructions. Real-Time PCR was performed in a ViiA7 System (Applied Biosystems) using Perfecta SYBR Green SuperMix Low Rox (Quanta). To determine the mRNA fold change of the different genes, the $\Delta \Delta \mathrm{Ct}$ quantitation method was used, with $36 B 4$ as the housekeeping gene. The primers used are listed in Supplemental Table 1. The TaqMan ${ }^{\circledR}$ Array, Human WNT Pathway, was used as instructed by the manufacturer (Applied Biosystems).

\section{Immunofluorescence}

Coverslips were rinsed with PBS and fixed in 4\% paraformaldehyde (Santa Cruz) for $20 \mathrm{~min}$, washed twice with PBS, permeabilized using $0.1 \%$ Triton in PBS for 15 min and washed 3 times with PBS to remove detergent. Blocking was performed using 2\% BSA, $50 \mathrm{mM}$ 
glycine, $0.01 \% \mathrm{NaN}_{3}$ in PBS for $1 \mathrm{~h}$. All primary antibodies were diluted in blocking solution and incubated overnight at $4^{\circ} \mathrm{C}$. Cells were then were washed gently 4 times with PBS, incubated with secondary antibody in the dark for $1 \mathrm{~h}$ (AlexaFluor488, AlexaFluor594 or AlexaFluor647, 1:500), washed 4 times, mounted using Vectashield (Vector Labs) and stored in the dark at $4^{\circ} \mathrm{C}$. Stained cells were visualized using a Leica Epifluorescence microscope and a Leica Confocal microscope. The primary antibodies used are listed in Supplemental Table 2. In order to quantify immunofluorescence, images were taken with the same exposure time consecutively without interruption using a Leica Epifluorescence microscope, and always handled as separate-channel images with ImageJ. A minimum and maximum intensity threshold was set to representatively measure the fluorescence for the channel of interest. These values were fixed for all images from the same experiment (4 random images per treatment), and the area covered by these intensities was measured and taken as the relative area of interest for each channel and image. The same was done for the DAPI channel for each image in order to obtain a ratio of the antibody and DAPI signals, which provides a measure of the stained area for that particular target protein. The 4 values per treatment were averaged and then the averages of three independent experiments were statistically processed.

\section{Gene reporter assays and gene silencing}

Cells plated in 12-well plates at 50\% confluence were washed twice with OptiMEM (Gibco) to remove antibiotics and transfected with $450 \mathrm{ng}$ luciferase gene reporters (Super8xTOPFlash and Super8xFOPFlash [27], AP-1-luciferase [28] and CRE-luciferase [29]), together with a constitutive Renilla luciferase reporter (pRL-tk) using Lipofectamine LTX and Plus Reagent (Invitrogen), as instructed by the supplier. The transfection mix was removed 4 hours later and fresh medium (with or without treatment) added. After 24-48 h, the cells were washed twice with PBS and lysed in 125 ul Passive Lysis Buffer (Promega). 
Luminescence was determined using the Dual-Glo Luciferase Assay System (Promega).

Signaling activities were calculated as luciferase/Renilla and TOP/FOP ratios.

Trisilencer-27 siRNA Knockdown Duplexes were obtained from OriGene (siCTRL, siATF2, siCREB, siJUN, siW3A); three siRNAs were tested for each gene (sequences are available on request). For FZD7 and VANGL2 ON-TARGETplus siRNA smartpools containing 4 different sequences were acquired from Dharmacon. Cells at 30-50\% confluence were washed twice in OptiMEM to remove antibiotics and transfected with siRNAs (25 nM for Trisilencer siRNAs and $100 \mathrm{nM}$ for smartpools) dissolved in OptiMEM using Lipofectamine RNAiMAX (Invitrogen), as described by the manufacturer. 24h after the addition of the transfection mix, the liposomes were removed and replaced with fresh medium.

\section{Western Blotting (WB)}

Total cell extracts were obtained by lysing the cells in radio-immunoprecipitation assay (RIPA) lysis buffer (Millipore) with 1 mM EDTA (Sigma), Complete Mini EDTA-free Protease Inhibitors (Roche), PhosphoStop (Roche) and 0.1\% SDS (Gibco) and centrifuged for $12 \mathrm{~min}$ at $15,000 \mathrm{~g}$. The supernatants were combined with $2 \mathrm{x}$ Laemmli Buffer (Sigma) and heated at $95^{\circ} \mathrm{C}$ or stored at $-80^{\circ} \mathrm{C}$. Nuclear and non-nuclear extracts were prepared using NEPER Nuclear and Cytoplasmic Extraction Kit (Thermo Scientific) with Complete Mini EDTA-free Protease Inhibitors (Roche). Proteins were separated on SDS polyacrylamide gels and transferred to PVDF membranes (Millipore). Membranes were washed in TBST (TBS $0.05 \%$ Tween (Sigma)) and incubated in blocking buffer (3\% BSA (Sigma) or 5\% milk in TBST) for $1 \mathrm{~h}$. Primary antibodies were incubated as described in Supplemental Table 3, washed 4 times in TBST, incubated for $1 \mathrm{~h}$ in blocking buffer with HRP-conjugated secondary antibodies diluted at 1:10,000, washed again and developed using chemiluminescence (Clarity Western ECL Substrate, BioRad).

\section{Statistics}


Three independent experiments were performed for each panel, unless otherwise stated.

Students $t$ test or ANOVA was applied to determine significance, as required. Error bars show SD or SEM.

\section{RESULTS}

\section{Neuronal differentiation of human neural progenitor cells correlates with a switch from Wnt/ק-catenin to noncanonical Wnt signaling.}

Characterization of hES-derived neural progenitor cells indicated that undifferentiated cells express the neural stem cell markers SOX2 and Nestin and do not express the pluripotency marker Nanog (Fig. S1A-C), confirming their commitment to the neuroectodermal lineage. Neuronal differentiation was monitored by induction of the early neuronal marker PAX6 at 1 day (Fig. S1F) and increasing expression of NEUROD1 (ND1, Fig. S1D) and $\beta$-III tubulin (Fig. S1C). In addition, we noted an increase in the intensity of $\beta$-catenin staining at the plasma membrane in differentiating cells (Fig. S1E). Wnt signaling activity in undifferentiated and differentiating hNP cells was then measured using gene reporter assays (Fig. 1). Wnt/ $\beta$-catenin signaling was significantly lower in differentiating cells, as indicated by a reduction in Super8xTOPFlash reporter activity; the control Super8xFOPFlash reporter with mutations in the Tcf/LEF binding sites was not affected (Fig. 1A). Noncanonical Wnt signals activate AP-1 transcription factors, particularly ATF2 [30, 31]. ATF2-luciferase provides a readout for noncanonical Wnt signaling in amphibians but not in mammalian cells [32]. However, AP-1 family transcription factor activity can be measured using AP-1- and CRE-luciferase reporters. Both AP-1- and CRE-luciferase activities increased upon neuronal differentiation (Fig. 1B, C). In addition, the phosphorylation and nuclear localization of ATF2 and CREB, which are induced by noncanonical Wnt signals in other cell types [31, 33, 34], 
increased during differentiation (Fig. 1D-G), whereas the level of nuclear $\beta$-catenin did not change (Fig. 1G). In summary, neural differentiation is accompanied by a reduction in Wnt/ßcatenin signaling and increases in AP-1- and CRE-luciferase activities and ATF2/cJun/CREB protein levels, suggestive of an increase in noncanonical Wnt signaling.

In order to determine if this apparent switch in Wnt signaling could be observed at the mRNA level, we examined expression of Wnt pathway components using a real-time PCR TaqMan array. Neuronal differentiation was verified by real-time PCR analysis of the expression of the neuronal genes NEUROD1 (ND1) and ASCL1 (Fig. S2A). The top twelve up- and down-regulated Wnt pathway genes included several noncanonical Wnts, whose expression increased during differentiation (Fig. S2B). There were fewer changes among the Wnt receptors, but FZD7 expression increased and FZD6 expression was reduced during differentiation. In addition, there was a reduction in the expression of the Wnt target genes NKD1, DKK1 and $M Y C$, but not of $A X I N 2$. Three independent time-course experiments using conventional real-time PCR were used to confirm these results (Fig. 2). There was a correlation between increased expression of the early neural genes ASCL1, ND1 and NGN2, increased expression of $W N T 3 A, W N T 4, W N T 5 A, W N T 5 B, W N T 7 A, W N T 7 B$ and $F Z D 7$ and reduced expression of FZD6, DKK1, MYC and NKD1 during differentiation (Fig. 2A-D). Since the upregulated Wnt genes predominantly activate the noncanonical pathway in neural cells $[9,35,36]$, these results are consistent with the gene reporter assays showing a reduction in $\mathrm{Wnt} / \beta$-catenin signaling and an increase in AP-1 signaling during differentiation.

\section{An endogenous noncanonical Wht signal is required for neuronal differentiation.}

In order to determine the importance of endogenous Wnts for neuronal differentiation, we tested the impact of blocking Wnt secretion using IWP-2, which inhibits porcupine, the enzyme that palmitoylates Wnt proteins [37]. Treatment of cells with IWP-2 reduced 


\section{Stem Cells}

Running title: Wnt signaling switch during neural differentiation

expression of neuronal differentiation markers both under basal conditions and during differentiation (Fig. 3A). The same results were obtained using a second porcupine inhibitor, Wnt-C59 [38] (Fig. 3C and D). IWP-2 reduced expression of the Wnt target genes AXIN2 and NKD1 in undifferentiated cells (Fig. 3B), indicating that they secrete and respond to canonical Wnts. In contrast, IWP-2 did not affect expression of AXIN2 or NKD1 in differentiating cells, consistent with their reduced canonical Wnt signaling activity. Importantly, IWP-2 reduced neuronal gene expression both in undifferentiated and differentiating cells, indicating that Wnt secretion is required for neuronal gene expression, irrespective of $\mathrm{Wnt} / \beta$-catenin signaling activity.

Since neuronal differentiation is often accompanied by glial differentiation, we also examined the expression of markers for astrocytic (CD44 and $S 100 A$ [39]) and oligodendrocytic (OLIG1 and PDGFRA [40]) differentiation. Wnt-C59 treatment of undifferentiated cells reduced expression of the astrocytic markers and increased expression of the oligodendrocytic markers (Fig. S2D), suggesting that endogenous Wnts affect basal expression of glial markers in undifferentiated cells. However, induction of hNP differentiation was accompanied by a strong reduction in the expression of these markers, indicating that these culture conditions promote neuronal rather than glial differentiation (Fig. S2C). Moreover, treatment of cells with Wnt-C59 did not affect glial marker expression in differentiating cells, as expected given the already reduced levels of these genes (Fig. S2D).

To determine if changes in $\mathrm{Wnt} / \beta$-catenin signaling directly affect neuronal differentiation, we used iCRT-14, which inhibits Wnt/ $\beta$-catenin signaling by blocking the interaction between $\beta$-catenin and TCF4 [41], and the GSK-3 inhibitor CHIR99021, which activates Wnt/ $\beta$ catenin signaling by preventing $\beta$-catenin degradation [42]. Treatment of cells with iCRT-14 and CHIR99021 induced a 2-fold reduction (Fig. 3E) and a 3-fold increase (Fig. 3F) in 
$\mathrm{Wnt} / \beta$-catenin activity, respectively. However, neither compound affected neuronal gene expression (Fig. 3G). Together, these results strengthen the case for noncanonical rather than $\mathrm{Wnt} / \beta$-catenin signaling playing a role in neuronal differentiation.

\section{Noncanonical Wht-3a signaling restores neuronal differentiation of hNP cells.}

To determine if exogenous Wnt proteins could rescue the effects of IWP-2, recombinant Wnts were added to IWP-2-treated hNP cells during differentiation. Wnt-3a, Wnt-7a and Wnt-5a (Fig. 4A-B and Fig. S2E) rescued neuronal gene expression in IWP-2-treated cells. Wnt-7a and Wnt-5a were much less effective than Wnt-3a, which completely restored neuronal gene expression to levels in differentiating control cells. This result was unexpected, since Wnt-3a normally strongly activates $\beta$-catenin, but is consistent with the increase in endogenous $W N T 3 A$ mRNA during differentiation (Fig. 2B). We therefore explored the function of Wnt-3a in more detail, hypothesizing that it rescues neuronal gene expression by activating a noncanonical signal. To test this possibility, we used Dkk-1, a secreted Wnt antagonist that binds to LRP5/6 Wnt co-receptors, thereby inhibiting canonical but not noncanonical Wnt signaling [1]. We surmised that if Wnt-3a rescues the effect of IWP-2 by activating a canonical Wnt signal, this would be prevented by Dkk-1 (Fig. S3A). As expected, Dkk-1 blocked activation of canonical signaling by Wnt-3a, as measured by inhibition of $\beta$ catenin/Tcf target gene expression (Fig. 4C). The results of gene reporter assays further demonstrated efficient inhibition of canonical Wnt signaling by Dkk-1 (Fig. S3B). However, Dkk-1 did not block the effects of Wnt-3a on neuronal gene expression (Fig. 4D), indicating that Wnt-3a promotes neuronal differentiation by activating a noncanonical signal. We also noted that differentiating cells formed islands of cells that express high levels of ND1 and 


\section{Stem Cells}

Running title: Wnt signaling switch during neural differentiation

doublecortin (DCX). Inhibition of Wnt secretion prevented formation of these islands, and this effect was rescued by Wnt-3a and by Wnt-3a and Dkk-1 together (Fig. 4E).

To extend these findings, we inhibited Wnt/ $\beta$-catenin signaling using IWR-1, which promotes $\beta$-catenin degradation by stabilizing Axin. As expected, IWR-1 inhibited Wnt-3a activation of $\beta$-catenin/Tcf transcriptional activity in Wnt-C59-treated cells (Fig. 5A) and Wnt/ $\beta$-catenin target gene expression (Fig. 5B). Consistent with the experiments using Dkk-1, IWR-1 did not affect neuronal gene expression (Fig. 5C). To determine if noncanonical Wnt3a signaling is sufficient for neuronal differentiation beyond the first 3 days, cells were treated for 7 days with Wnt-3a and the same inhibitors. We then analyzed neuronal differentiation by q-PCR (Fig. 5D) and by immunofluorescence for DCX and $\beta$-III tubulin (Fig. 5E-G). Wnt-C59 inhibition of Wnt secretion completely blocked neuronal differentiation and this was rescued by recombinant Wnt-3a in the presence of IWR-1, supporting a role for noncanonical Wnt-3a signaling at later stages of differentiation.

\section{Wnt-3a/AP-1 signaling is required for neuronal differentiation.}

To determine if noncanonical Wnt-3a signals increase neuronal differentiation via AP-1 signaling, we examined the effects of Wnt-C59 on AP-1 family proteins in differentiating cells. hNP cells cultured in the presence of Wnt-C59 and treated with Wnt-3a with or without Dkk-1 were analyzed for AP-1 family proteins using immunofluorescence. Wnt-3a increased the levels of phospho-CREB, phospho-ATF2, ATF2 and c-Jun in differentiating islands of cells, and these changes were not affected by Dkk-1 (Fig. S4A-D). This further supports a model in which Wnt-3a/AP-1 rather than Wnt-3a/ $\beta$-catenin signaling promotes neuronal differentiation. 
JNK is a kinase implicated in noncanonical Wnt signaling that can phosphorylate and stabilize ATF2 and c-Jun. To determine if JNK signaling is required for neuronal differentiation, we tested the effects of the JNK-specific inhibitor JI8 [43]. JI8 reduced the levels of c-Jun, phospho-CREB, phospho-ATF2 and ATF2 (Fig. 6A), AP-1 gene reporter activity (Fig. S5A) and expression of neuronal genes (Fig. 6B). To determine if JNK activity is required for Wnt-3a induction of neuronal differentiation independently of Wnt-3a/ $\beta$ catenin signaling, we tested the effects of JI8 using cells cultured in Wnt-C59 and treated with Wnt-3a and IWR-1. JI8 was found to inhibit neuronal gene expression (Fig. 6C) without affecting Wnt/ $\beta$-catenin target gene expression (Fig. 6D), consistent with a model in which Wnt-3a/JNK signaling promotes neuronal differentiation independently of Wnt/ $\beta$-catenin.

We next used gene silencing to determine if any of the AP-1 family proteins are specifically required for neuronal differentiation. In addition, since $W N T 3 A$ mRNA expression was observed to increase early during differentiation and from a lower baseline than other Wnt family members, we also silenced Wnt-3a. Optimal siRNAs for silencing ATF2, CREB, JUN and WNT3A were selected (Fig. 6G). Silencing Wnt-3a and ATF2, but not CREB or JUN, reduced neuronal gene and protein expression in differentiating cultures (Fig. 6E and G and S6B-C), highlighting the importance of endogenous Wnt-3a and ATF2 in neuronal differentiation of hNP cells. In order to determine if ATF2 is required for Wnt-3a restoration of neuronal differentiation, cells were transfected with siRNAs, treated with Wnt-C59 to block endogenous Wnt secretion and treated with Wnt-3a in the presence of IWR-1 to selectively activate noncanonical Wnt signaling. Silencing of ATF2 reduced expression of three of the four neuronal genes tested (Fig. 6F), indicating that ATF2 is an important downstream effector of Wnt-3a during neuronal differentiation. 


\section{Noncanonical Wnt-3a and ATF2 promote neuronal differentiation in iPS-derived neural progenitor cells.}

In order to determine if Wnt-3a/AP-1 signaling is of general importance for neuronal differentiation of human stem cells, we carried out studies using human iPS cell-derived neural progenitor cells. The cells were first evaluated by immunocytochemistry and western blotting, which confirmed that they express the neural stem cell markers SOX2 and Nestin and can be induced to undergo neuronal differentiation, as indicated by expression of DCX and PAX6, which was observed at 5 days (Fig. S6A-D). We also observed increased levels of phospho-ATF2 in differentiating cells (Fig. S6C). Neuronal gene expression was measured in a 7-day time course to provide an indication of the rate of neuronal differentiation (Fig. S7A). Consistent with what was seen in hNP cells, neuronal differentiation was accompanied by increased expression of $W N T 3 A, W N T 7 A$ and $W N T 7 B$ (Fig. S7B), reduced expression of $D K K 1$ and $N K D 1$, and a small increase in $A X I N 2$ expression (Fig. S7C). As we previously observed in hNP cells, glial markers were also reduced upon differentiation (Fig. S7D).

Given the similarities with hNP cells, we examined the responses of iPS cell-derived neural progenitor cells to Wnt-C59 and subsequent treatment with Wnt-3a in the presence or absence of IWR-1 to block Wnt/ $\beta$-catenin signaling. Wnt-C59 inhibited neuronal differentiation of iPS cell-derived neural progenitors, as measured by expression of DCX in cell cultures (Fig. 7A, B) and by expression of neuronal genes. Addition of recombinant Wnt-3a rescued neuronal gene expression and IWR-1 was unable to block this effect of Wnt-3a (Fig. 7C), although it did inhibit Wnt-3a-induced expression of Wnt/ $\beta$-catenin target genes (Fig. S7E). The levels of phospho-ATF2 responded similarly to these treatments; being reduced by Wnt-C59 and stimulated by Wnt-3a in the presence or absence of IWR-1 (Fig. 7F). Finally, ATF2 gene silencing reduced the number of DCX-positive cells in differentiating cultures (Fig. 7D and E), indicating that ATF2 is also important for differentiation of iPS-derived neural 
progenitors. These results are similar to those obtained using hNP cells, suggesting Wnt3a/ATF2 signaling plays a general role in neural stem cell differentiation.

\section{DISCUSSION}

The demonstration that inhibition of Wnt secretion using the porcupine inhibitors IWP-2 and Wnt-C59 blocks neural stem cell differentiation is consistent with studies highlighting the importance of Wnt signaling in neural development [44]. However, our observations that $\beta$ catenin/Tcf activity and target gene expression fall during differentiation, and that activation and inhibition of $\mathrm{Wnt} / \beta$-catenin signaling do not affect neuronal differentiation, are not in keeping with a role for $\mathrm{Wnt} / \beta$-catenin signaling in differentiation per se. Inhibition of $\mathrm{Wnt}$ secretion had different effects on $\beta$-catenin/Tcf target gene expression in undifferentiated and differentiating cells, consistent with $\mathrm{Wnt} / \beta$-catenin signaling playing a role in self-renewal and/or survival of neural progenitors, rather than differentiation $[10,11]$. However, we cannot rule out that $\mathrm{Wnt} / \beta$-catenin signaling during the self-renewal phase also primes the cells to differentiate appropriately. We also tried to determine whether Wnt signaling was implicated in the differentiation of glial cells. Inhibition of Wnt secretion in undifferentiated cells did alter basal expression of glial genes, favoring expression of oligodendrocytic over astrocytic markers. However, culture in neuronal differentiation medium reduced basal expression of glial markers both in hNP and iPS-derived NSCs, preventing further analysis of the effects of noncanonical Wnt signaling on glial differentiation

Several Wnt genes were upregulated during neuronal differentiation. Among these, WNT3A, $W N T 7 A$ and $W N T 7 B$ were strongly induced in both hNP and iPS-derived neural progenitor cells. Wnt-3a, in particular, appears to play a key role, since gene silencing of Wnt-3a alone inhibited neuronal gene expression and recombinant Wnt-3a was sufficient to restore neuronal differentiation in cells treated with porcupine inhibitors. The patterns of expression of these 
Wnts suggest they play a role in mouse neural progenitors, with $W N T 3 A$ being found in the ventricular zone of the dorsal spinal cord and the hindbrain [45] and $W N T 7 A$ and $W N T 7 B$ present in the ventricular zone in the ventral-lateral spinal cord and cortical forebrain [45], and $W N T 7 B$ also expressed in the adult dentate gyrus (Fig. S8A, Supplemental online video 1) (http://mouse.brain-map.org [46]). Wnt-7a and Wnt-5a were less efficient than Wnt-3a at restoring neuronal differentiation in hNP cells. Nevertheless, both Wnt-7a and Wnt-5a have been demonstrated to activate noncanonical signals in neurons: Wnt-7a, for example, promotes dendritic spine growth and synaptic strength in hippocampal neurons [35], while Wnt-5a is implicated in the morphogenesis and neurogenesis of dopaminergic neurons [17]. Whether Wnt-5a and Wnt-7a/b play distinct roles in NSC differentiation, compared to Wnt3a, remains to be determined.

Our results indicate that the noncanonical Wnt signal that drives neuronal differentiation involves JNK and AP-1 family members. As noted in the Introduction, there are several different noncanonical Wnt signaling pathways. In order to begin to address which of them might be involved in the response to Wnt-3a, we used a number of other small molecule inhibitors, but the results were inconclusive: inhibition of Rho kinase using Y-27632 did not affect expression of $N G N 2$ or $D C X$ and increased $N D 1$ expression, while inhibition of CaMKII using Myr-AIP slightly reduced NGN2 and DCX expression and did not affect ND1 (Fig. S9C-D). In addition, silencing of the Wnt/PCP pathway component VANGL2, which was upregulated during differentiation, did not significantly affect neuronal gene expression (Fig. S9A-B). While these results do not exclude the involvement of Wnt/Calcium and Wnt/PCP signaling, the lack of consistent effects prompted us to focus on JNK/AP-1. Wnt-3a, Wnt-7a and Wnt-7b have all been shown to activate JNK [36, 47-49], which is a major AP-1 kinase [50]. Moreover, inhibition of JNK blocked the ability of Wnt-3a to restore neuronal differentiation. Neuronal differentiation of hNP cells was paralleled by increases in AP-1- and 
CRE-dependent gene reporter activities and expression and/or phosphorylation of the ATF2, c-Jun and CREB. Both ATF2 and CREB have been linked to neurogenesis in the adult mouse hippocampus $[18,51]$, and, according to data from the Allen Brain Atlas, both are highly expressed in the dentate gyrus (Fig. S8B, C and supplemental online video 2 (Atf2)), supporting a role for these proteins in differentiation of adult NSCs in vivo. Our gene silencing experiments indicate that ATF2 is required for neuronal gene expression in both $\mathrm{hNP}$ and iPS-derived neural progenitor cells. However, there is considerable crosstalk among AP-1 family members, so roles for c-Jun and CREB should not be ruled out.

Since Wnt-3a signals can both maintain mouse neural stem/progenitor cells [52] and promote neural differentiation [53], the response to Wnt-3a is likely to depend on the Wnt receptors expressed and/or the presence of other factors that affect Wnt signaling, such as secreted Wnt antagonists. Dkk-1, for example, has been reported to suppress neural progenitor proliferation [11] and sFRP3 inhibits quiescent NSC activation and neural progenitor maturation [54]. However, abrogation of sFRP3, but not Dkk-1 expression, increases the number of mature neurons. This supports a model in which noncanonical Wnt signals play a role in neuronal differentiation, since only sFRP3 inhibits both canonical and noncanonical signaling. We have used Dkk-1 as a tool to block canonical Wnt signaling and thereby reveal the noncanonical signaling function of Wnt-3a. We obtained similar results using IWR-1 to inhibit canonical signaling both in hNP and iPS-derived neural progenitor cells, further supporting the view that $\beta$-catenin signaling activity is not required for the effect of Wnt-3a. Noncanonical Wnt-3a signaling has been observed in mesenchymal stem cells, where it inhibits chondrogenesis via activation of calcium/calmodulin-dependent kinase II (CaMKII) [55], and in Ewing tumor cells, where Wnt-3a and Dkk-1 induce neurite outgrowth [47]. However, to our knowledge, this is the first report describing a noncanonical role for Wnt-3a in hES and iPS-derived neural progenitor cells. 


\section{Stem Cells}

Running title: Wnt signaling switch during neural differentiation

The mechanism responsible for the switch in the response to Wnt-3a remains unclear.

Differentiation is initiated by the removal of FGF2. FGF2 has been reported to increase canonical Wnt signaling via inhibition of GSK-3 [56], so its removal may be responsible for the observed reduction in $\mathrm{Wnt} / \beta$-catenin signaling. The switch to noncanonical signaling could result from changes in expression of inhibitors or effectors of Wnt canonical and noncanonical signaling. Neuronal differentiation of hNP cells is accompanied by a fall in expression of both $D K K 1$ and $D K K 2$, so Dkk proteins are unlikely to be involved. One possibility is that it is mediated by changes in Wnt receptor expression. Our analysis of Wnt receptor mRNA expression levels did not reveal many changes apart from increased expression of FZD7 (Fig. 2Cand S2B). However, when we silenced FZD7 there was no effect on neuronal gene expression (Fig. S9A-B). A complete answer will require silencing combinations of candidate Wnt receptors. In addition, differentiation may be accompanied by posttranscriptional changes in the Wnt receptor repertoire, a prospect that deserves further investigation.

Another possibility is that the switch involves changes in the relative expression levels of Tcf/LEF and AP-1 family transcription factors. A recent report showed that RA-induced differentiation of mES cells is accompanied by a reduction in Tcf- 1 and an increase in Tcf-3 expression, with the latter repressing canonical Wnt signaling and promoting noncanonical signaling and differentiation [57]. We did not observe changes in the expression of TCF (Tcf1) in the Wnt array analysis, but there was a 1.7-fold increase in expression of TCF7L1 (Tcf3) upon differentiation (unpublished observations), so it is possible that Tcf-3 plays a role in the switch. Alternatively, the switch could result from increased expression of AP-1 family members, particularly ATF2. ATF2 has been reported to bind to Tcf family proteins in hematopoietic tumor cells, leading to activation of a Tcf reporter [58]. While this may be inconsistent with the reduction in Tcf reporter activity we observed in differentiating neural 
stem cells, the transcriptional activity of ATF2/Tcf complexes may be context-dependent.

Given the therapeutic potential of human neural stem cells, future studies directed at clarifying the mechanism responsible for the switch may prove fruitful.

\section{Conclusion}

In summary, our results indicate that neuronal differentiation is accompanied by a reduction in canonical Wnt signaling, and, in addition, highlight the importance of noncanonical Wnt signaling in this process in two independent stem cell models. Manipulation of Wnt signaling with purified Wnt proteins is used for generating neurons [17] and other cell types [59] from stem cells. A better understanding of how the cellular response to Wnt proteins switches from canonical to noncanonical during differentiation may therefore be important for advancing stem cell-based cell replacement therapies.

\section{Acknowledgements}

We thank Victor Campa and Mercedes Caro for their training and unconditional support. We also thank Randall Moon (University of Washington, Seattle) for pSuper8XTOPflash and pSuper8XFOPflash, Jan Brosens (Warwick University, UK) for CRE-luciferase and Maria Vivanco (CIC bioGUNE) for AP-1-luciferase.

\section{Disclosure of potential conflicts of interest}

The authors declare they have no competing interests. 


\section{References}

1. MacDonald BT, Tamai K, He X. Wnt/beta-catenin signaling: components, mechanisms, and diseases. DEV. CELL 2009;17(1):9-26.

2. Clevers H, Nusse R. Wnt/ $\beta$-Catenin Signaling and Disease. CELL 2012;149(6):1192-1205.

3. Amerongen R Van, Nusse R. Towards an integrated view of Wnt signaling in development. DEV. CAMBRIDGE ENGL. 2009; 136(19):3205-3214.

4. Lopez-Bergami P, Lau E, Ronai Z. Emerging roles of ATF2 and the dynamic AP1 network in cancer. NAT. REV. CANCER 2010;10(1):65-76.

5. Clark CEJ, Nourse CC, Cooper HM. The tangled web of non-canonical Wnt signalling in neural migration. NEUROSIGNALS. 2012;20(3):202-20.

6. Yu H, Ye X, Guo N, et al. Frizzled 2 and frizzled 7 function redundantly in convergent extension and closure of the ventricular septum and palate: evidence for a network of interacting genes. DEVELOPMENT 2012;139(23):4383-94.

7. Najdi R, Proffitt K, Sprowl S, et al. A uniform human Wnt expression library reveals a shared secretory pathway and unique signaling activities. DIFFER. RES. BIOL. DIVERS. 2012:1-11.

8. Lange C, Mix E, Frahm J, et al. Small molecule GSK-3 inhibitors increase neurogenesis of human neural progenitor cells. NEUROSCI. LETT. 2011;488(1):36-40.

9. Elizalde C, Campa VM, Caro M, et al. Distinct roles for Wnt-4 and Wnt-11 during retinoic acid-induced neuronal differentiation. STEM CELLS 2011;29(1):141-153.

10. Bei-Yu C, Wang X, Wang Z-Y, et al. Brain-derived neurotrophic factor stimulates proliferation and differentiation of neural stem cells, possibly by triggering the $\mathrm{Wnt} / \mathrm{B}$-catenin signaling pathway. $\mathbf{J}$ NEUROSCI RES 2013;91(1):30-41.

11. Seib D, Corsini N, Ellwanger K, et al. Loss of dickkopf-1 restores neurogenesis in old age and counteracts cognitive decline. CELL STEM CELL 2013;12(2):204-14.

12. Pöschl J, Grammel D, Dorostkar M, et al. Constitutive activation of $\beta$-catenin in neural progenitors results in disrupted proliferation and migration of neurons within the central nervous system. DEV. BIOL. 2013;374(2):319-32.

13. Imura T, Wang X, Noda $\mathrm{T}$, et al. Adenomatous polyposis coli is essential for both neuronal differentiation and maintenance of adult neural stem cells in subventricular zone and hippocampus. STEM CELLS 2010;28(11):2053-2064.

14. Wexler EM, Paucer A, Kornblum HI, et al. Endogenous Wnt signaling maintains neural progenitor cell potency. STEM CELLS 2009;27(5):1130-41.

15. Nicoleau C, Varela C, Bonnefond C, et al. ES Cells Neural Differentiation Qualifies the Role of Wnt/ $\beta$ Catenin Signals in Human Telencephalic Specification and Regionalization. STEM CELLS 2013:N/AN/A.

16. Varela-Nallar L, Inestrosa NC. Wnt signaling in the regulation of adult hippocampal neurogenesis. FRONT. CELL. NEUROSCI. 2013;7(June):100.

17. Andersson ER, Saltó C, Villaescusa JC, et al. Wnt5a cooperates with canonical Wnts to generate midbrain dopaminergic neurons in vivo and in stem cells. PROC. NATL. ACAD. SCI. 2013;110 (7 ):E602-E610.

18. Pearson AG, Curtis MA, Waldvogel HJ, et al. Activating transcription factor 2 expression in the adult human brain: association with both neurodegeneration and neurogenesis. NEUROSCIENCE 2005;133(2):437451.

19. Merz K, Herold S LD. CREB in adult neurogenesis--master and partner in the development of adult-born neurons? EUR. J. NEUROSCI. 2011;33(6):1078-86.

20. Aimone JB, Deng W, Gage FH. Adult neurogenesis: integrating theories and separating functions. TRENDS COGN. SCI. 2010;14(7):325-337.

21. Lie D-C, Colamarino S a, Song H-J, et al. Wnt signalling regulates adult hippocampal neurogenesis. NATURE 2005;437(7063):1370-5.

22. Zhao C, Deng W, Gage FH. Mechanisms and functional implications of adult neurogenesis. CELL 2008;132(4):645-660.

23. Wichterle H, Przedborski S. What can pluripotent stem cells teach us about neurodegenerative diseases[quest]. NAT. NEUROSCI. 2010;13(7):800-804.

24. Stice SKD and SL. Neural Differentiation of Human Embryonic Stem Cells. J. CELL. BIOCHEM. 2008;105:633-640.

25. Erceg S, Ronaghi M, Stojković M. Human embryonic stem cell differentiation toward regional specific neural precursors. STEM CELLS 2009;27(1):78-87. 
26. Imaizumi Y, Okano H. Modeling human neurological disorders with induced pluripotent stem cells. J. NEUROCHEM. 2013:1-12.

27. Veeman MT, Slusarski DC, Kaykas A, et al. Zebrafish Prickle, a Modulator of Noncanonical Wnt / Fz Signaling, Regulates Gastrulation Movements. CURR. BIOL. 2003;13:680-685.

28. Vivanco MD, Johnson R, Galante PE, et al. A transition in transcriptional activation by the glucocorticoid and retinoic acid receptors at the tumor stage of dermal fibrosarcoma development. EMBO J. 1995;14(10):2217-28.

29. Gene P, Stromal E, Telgmann R, et al. Activated Protein Kinase A Is Required for Differentiation-Dependent Transcription of the Decidual. ENDOCRINOLOGY 1997;138(3).

30. Zhou W, Lin L, Majumdar A, et al. Modulation of morphogenesis by noncanonical Wnt signaling requires ATF/CREB family-mediated transcriptional activation of TGFbeta2. NAT. GENET. 2007;39(10):12251234.

31. Schambony A, Wedlich D. Wnt-5A/Ror2 regulate expression of XPAPC through an alternative noncanonical signaling pathway. DEV. CELL 2007;12(5):779-92.

32. Ohkawara B, Niehrs C. An ATF2-based luciferase reporter to monitor non-canonical Wnt signaling in Xenopus embryos. DEV. DYN. AN OFF. PUBL. AM. ASSOC. ANAT. 2011;240(1):188-194.

33. Kohn AD, Moon RT. Wnt and calcium signaling: beta-catenin-independent pathways. CELL CALCIUM 2005;38(3-4):439-446.

34. Chen AE, Ginty DD, Fan C-M. Protein kinase A signalling via CREB controls myogenesis induced by Wnt proteins. NATURE 2005;433(7023):317-22.

35. Ciani L, Boyle K. Wnt7a signaling promotes dendritic spine growth and synaptic strength through Ca2+/Calmodulin-dependent protein kinase II. PROC. ... 2011.

36. Rosso SB, Sussman D, Wynshaw-Boris A, et al. Wnt signaling through Dishevelled, Rac and JNK regulates dendritic development. NAT. NEUROSCI. 2005;8(1):34-42.

37. Chen B, Dodge ME, Tang W, et al. Small molecule-mediated disruption of Wnt-dependent signaling in tissue regeneration and cancer. NAT. CHEM. BIOL. 2009;5(2):100-107.

38. Proffitt K, Madan B, Ke Z, et al. Pharmacological inhibition of the Wnt acyltransferase PORCN prevents growth of WNT-driven mammary cancer. CANCER RES. 2013;73(2):502-7.

39. Majumder A, Dhara SK, Swetenburg R, et al. Inhibition of DNA methyltransferases and histone deacetylases induces astrocytic differentiation of neural progenitors. STEM CELL RES. 2013;11(1):574-86.

40. Alsanie WF, Niclis JC, Petratos S. Human embryonic stem cell-derived oligodendrocytes: protocols and perspectives. STEM CELLS DEV. 2013;22(18):2459-76.

41. Gonsalves FC, Klein K, Carson BB, et al. An RNAi-based chemical genetic screen identifies three smallmolecule inhibitors of the Wnt/wingless signaling pathway. PROC. NATL. ACAD. SCI. U. S. A. 2011;108(15):5954-5963.

42. Wagman AS, Johnson KW, Bussiere DE. Development of 11beta-HSD1 inhibitors for the treatment of type 2 diabetes. CURR. PHARM. DES. 2004;10(10):702-710.

43. Szczepankiewicz BG, Kosogof C, Nelson LTJ, et al. Aminopyridine-Based c-Jun N-Terminal Kinase Inhibitors with Cellular Activity and Minimal Cross-Kinase Activity. J. MED. CHEM. 2006;29(Scheme 1):3563-3580.

44. Tabar V, Studer L. Pluripotent stem cells in regenerative medicine: challenges and recent progress. NAT. REV. GENET. 2014;15:82-92.

45. Daneman R, Zhou L, Kuhnert F, et al. 'Wnt/ NL -catenin signaling is required for CNS, but not non-CNS, angiogenesis. PROC. NATL. ACAD. SCI. U. S. A. 2009;106(2):641-646.

46. Lein ES, Hawrylycz MJ, Ao N, et al. Genome-wide atlas of gene expression in the adult mouse brain. NATURE 2007;445(7124):168-76.

47. Endo Y, Beauchamp E, Woods D, et al. Wnt-3a and Dickkopf-1 Stimulate Neurite Outgrowth in Ewing Tumor Cells via a Frizzled3- and c-Jun N-Terminal Kinase-Dependent Mechanism. MOL. CELL. BIOL. 2008;28(7):2368-2379.

48. Bikkavilli RK, Feigin ME, Malbon CC. p38 mitogen-activated protein kinase regulates canonical Wnt-betacatenin signaling by inactivation of GSK3beta. J. CELL SCI. 2008;121(Pt 21):3598-607.

49. Winn R a, Marek L, Han S-Y, et al. Restoration of Wnt-7a expression reverses non-small cell lung cancer cellular transformation through frizzled-9-mediated growth inhibition and promotion of cell differentiation. J. BIOL. CHEM. 2005;280(20):19625-34.

50. Bogoyevitch M a., Kobe B. Uses for JNK: the Many and Varied Substrates of the c-Jun N-Terminal Kinases. MICROBIOL. MOL. BIOL. REV. 2006;70(4):1061-1095.

51. Mantamadiotis T, Papalexis N, Dworkin S. CREB signalling in neural stem/progenitor cells: Recent developments and the implications for brain tumour biology. BIOESSAYS 2012;34(4):293-300. 
52. Kalani MYS, Cheshier SH, Cord BJ, et al. Wnt-mediated self-renewal of neural stem/progenitor cells. PROC. NATL. ACAD. SCI. U. S. A. 2008;105(44):16970-16975.

53. Muroyama Y, Kondoh H, Takada S. Wnt proteins promote neuronal differentiation in neural stem cell culture. BIOCHEM. BIOPHYS. RES. COMMUN. 2004;313(4):915-921.

54. Jang $\mathrm{M}-\mathrm{H}$, Bonaguidi $\mathrm{M}$ a, Kitabatake $\mathrm{Y}$, et al. Secreted frizzled-related protein 3 regulates activitydependent adult hippocampal neurogenesis. CELL STEM CELL 2013;12(2):215-23.

55. Nalesso G, Sherwood J, Bertrand J, et al. WNT-3A modulates articular chondrocyte phenotype by activating both canonical and noncanonical pathways. J. CELL BIOL. 2011;193(3):551-64.

56. Ding VMY, Ling L, Natarajan S, et al. FGF-2 modulates Wnt signaling in undifferentiated hESC and iPS cells through activated PI3-K/GSK3beta signaling. J. CELL. PHYSIOL. 2010;225(2):417-428.

57. Osei-Sarfo K, Gudas LJ. Retinoic acid suppresses the canonical Wnt signaling pathway in embryonic stem cells and activates the noncanonical Wnt signaling pathway. STEM CELLS 2014:n/a-n/a.

58. Grumolato L, Liu G, Haremaki T, et al. $\beta$-Catenin-independent activation of TCF1/LEF1 in human hematopoietic tumor cells through interaction with ATF2 transcription factors. PLOS GENET. 2013;9(8):e1003603.

59. Hay DC, Fletcher J, Payne C, et al. Highly efficient differentiation of hESCs to functional hepatic endoderm requires ActivinA and Wnt3a signaling. PROC. NATL. ACAD. SCI. 2008;105 (34 ):12301-12306. 


\section{Figure legends}

Fig. 1. A switch from canonical to noncanonical Wnt signaling in hNP cells. (A-C) Gene reporter assays measuring $\beta$-catenin/Tcf- (A), AP-1- (B) and CRE- (C) dependent transcriptional activities in undifferentiated and differentiating cells at $48 \mathrm{~h} ;{ }^{*} \mathrm{p}<0.05$ by $t$ test (error bars represent SD). (D-F) Immunofluorescence staining for phospho-ATF2 (D), ATF2 (E) and c-Jun (F) in undifferentiated and differentiating hNP cells; DAPI staining of nuclei is in blue. Scale bars 100 um. (G) Western blots for the indicated antigens in nuclear (NUC) and cytoplasmic (CYT) extracts from hNP cells at 1, 2 and 3 days of differentiation; ß-cat top short exposure, bottom long exposure; lamin A (LAM A) and HSP60 were used as loading controls.

Fig. 2. Neuronal and Wnt pathway gene expression during neuronal differentiation of hNP cells. Graphs show average mRNA expression determined by q-PCR of the genes indicated, relative to $36 \mathrm{~B} 4$, from 3 independent 5 day time-course experiments (error bars represent SEM). WNT3A mRNA could not be detected at day 0 , therefore graph B is normalized to day 1 whereas $\mathrm{A}, \mathrm{C}$ and $\mathrm{D}$ are normalized to day 0 .

Fig. 3. Inhibition of Wnt secretion blocks neuronal differentiation while inhibition of Wnt/ $\beta$-catenin signaling does not. (A, B) mRNA expression determined by q-PCR of the genes indicated, using undifferentiated hNP cells (UNDIF) or cells differentiated for $3 \mathrm{~d}$ (DIF) in the presence of vehicle (DMSO) or IWP-2 $(2 \mathrm{uM})$. (C, D) mRNA expression determined as in (A) in undifferentiated (UNDIF) or differentiating (DIF) cells treated with vehicle or the indicated concentrations of Wnt-C59. (E, F) Gene reporter assays measuring $\beta$ catenin/Tcf-dependent transcriptional activity (TOPFlash/FOPFlash ratio) in differentiating hNP cells treated with CHIR (E) or iCRT14 (F) for 24 h. (G) mRNA expression determined by q-PCR of the genes indicated, using differentiating cells treated with vehicle (DMSO), 
CHIR99021 (CHIR, $0.5 \mathrm{uM})$ and iCRT14 (1 uM). (A, B, E and F) ${ }^{*} \mathrm{p}<0.05$ by $t$ test, $(\mathrm{C}, \mathrm{D}$ and $\mathrm{G}) * \mathrm{p}<0.05$ by ANOVA (error bars represent $\mathrm{SD}$ ).

Fig. 4. Noncanonical Wnt-3a promotes early neuronal differentiation. (A, B) mRNA expression determined by q-PCR using differentiating cells treated with vehicle (DMSO and CHAPS), IWP2 and Wnt-7a (400 ng/ml) (A) or Wnt-3a (200 ng/ml) (B). (C, D) mRNA expression determined by q-PCR of the genes indicated, using differentiating hNP cells treated as indicated with vehicle (DMSO and CHAPS), IWP2 (2 uM), Wnt-3a (100 ng/ml) and/or Dkk-1 (100 ng/ml). (E) Immunofluorescence staining for DCX and ND1 in differentiating cells treated Wnt-C59 (100 nM) and vehicle (CHAPS), recombinant Wnt-3a (100 ng/ml), recombinant Dkk-1 (100 ng/ml) and vehicle (CHAPS) or both together; DAPI staining of nuclei is in blue, scale bars 200 um. ${ }^{*} \mathrm{p}<0.05$ by ANOVA (error bars represent $\mathrm{SD})$.

Fig. 5. Noncanonical Wnt-3a promotes neuronal differentiation at 7 days of differentiation. (A) $\beta$-catenin/Tcf activity in cells treated for $24 \mathrm{~h}$ with Wnt-C59 and vehicle, IWR-1 and/or Wnt-3a. (B, C) mRNA expression in differentiating cells cultured in Wnt-C59 and treated with vehicle, IWR-1 (10 uM) and/or Wnt-3a (100 ng/ml). (D) mRNA expression in differentiating cells cultured in vehicle, Wnt-C59 (100 nM) and vehicle, Wnt-C59 (100 nM), Wnt-3a (100 ng/ml) and vehicle, and Wnt-C59 (100 nM), Wnt-3a (100 ng/ml) and IWR$1(10 \mathrm{uM})$ for 7 days. (E) Immunofluorescence staining for DCX and $\beta$-III tubulin in differentiating cells treated for 7 days with vehicle (DMSO and CHAPS), Wnt-C59 (100 nM) and vehicle (CHAPS), Wnt-C59 (100 nM) and recombinant Wnt-3a (100 ng/ml), and WntC59 (100 nM), recombinant Wnt-3a (100 ng/ml) and IWR (10 uM); DAPI staining of nuclei is in blue; scale bars 200 um. (F, G) Relative quantification of DCX and $\beta$-III tubulin; * $p<$ 0.05 by ANOVA (error bars represent SD). 
Fig. 6. Wnt-3a restoration of neuronal differentiation is blocked by inhibition of JNK and by silencing of ATF2. (A) Western blots detecting the indicated antigens in total extracts of hNP cells cultured with vehicle (DMSO) or JNK inhibitor JI8 (10 uM); GAPDH and HSP60 were used as loading controls. (B) mRNA expression determined by q-PCR of the genes indicated, using differentiating cells treated with vehicle or JI8. (C, D) mRNA expression determined as in (B) using differentiating cells treated with Wnt-C59 (100 nM), IWR-1 (10 uM), Wnt-3a (100 ng/ml) and vehicle or JI8; * $\mathrm{p}<0.05$ by $t$ test (error bars represent $\mathrm{SD}$ ). (E, G) mRNA expression determined as in (B) and western blots using differentiating cells silenced for $72 \mathrm{~h}$ and compared to their respective controls depicted as a dashed line set at $1 ; * \mathrm{p}<0.05$ by $t$ test (error bars represent $\mathrm{SD}$ ). (F) mRNA expression using differentiating cells transfected with control (siC) or ATF2 siRNAs for $48 \mathrm{~h}$ and treated with Wnt-C59 (100 nM), IWR-1 (10 uM) and Wnt-3a (100 ng/ml) for a further 48h; * $\mathrm{p}<0.05$ by $t$ test (error bars represent SD).

Fig. 7. Noncanonical Wnt-3a and ATF2 promote neuronal differentiation in iPS-derived neural progenitor cells. (A) Immunofluorescence staining for DCX in differentiating cells treated for 5 days with vehicle (DMSO and CHAPS), Wnt-C59 (100 nM) and vehicle (CHAPS), Wnt-C59 (100 nM) and recombinant Wnt-3a (100 ng/ml), and Wnt-C59 (100 nM), recombinant Wnt-3a (100 ng/ml) and IWR (10 uM); DAPI staining of nuclei is in blue; scale bars 200 um. (B) Relative quantification of DCX levels; * $\mathrm{p}<0.05$ by $t$ test (error bars represent SEM). (C) mRNA expression determined by q-PCR of the genes indicated, using differentiating cells treated as indicated in $(\mathrm{A}) ;{ }^{*} \mathrm{p}<0.05$ by ANOVA test (error bars represent SD). (D) Immunofluorescence staining for DCX in differentiating cells silenced with control (siC) or ATF2 siRNA for 5 days; DAPI staining of nuclei is in blue; scale bars 200 um. (E) Relative quantification of DCX levels; * $\mathrm{p}<0.05$ by $t$ test (error bars represent 
SD). (F) Immunofluorescence staining of P-ATF2 in differentiating cells treated as indicated in (A). 
Bengoa, FIG.1
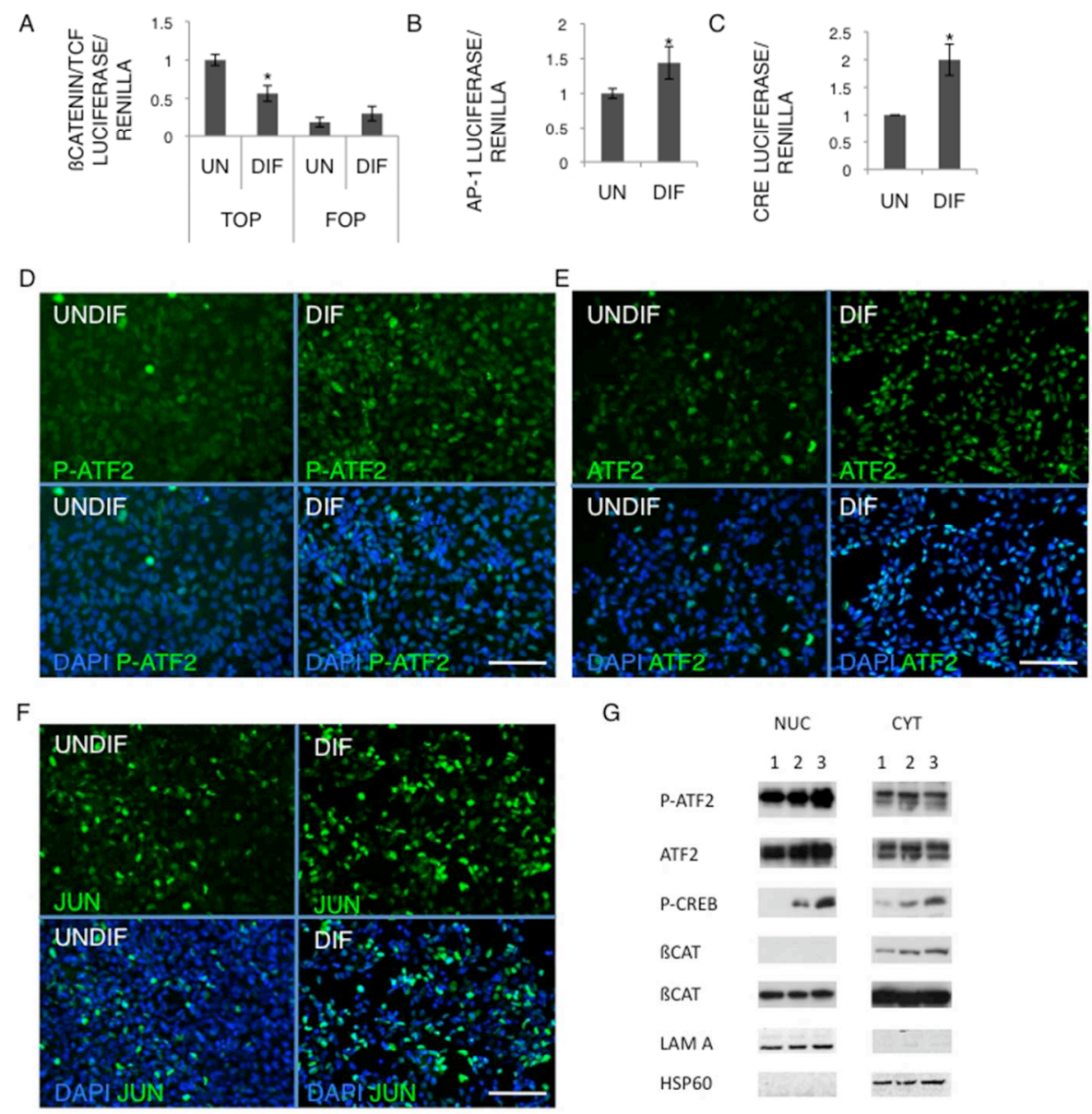

G

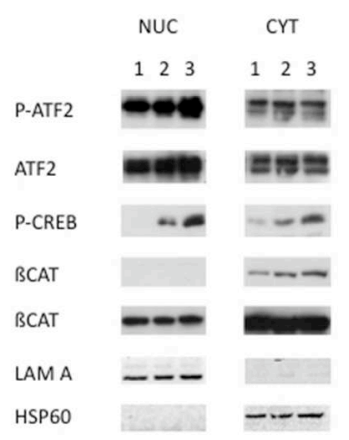

$264 \times 352 \mathrm{~mm}(72 \times 72$ DPI $)$

ScholarOne Support: (434) 964-4100 


\section{Stem Cells}

Bengoa, FIG.2

A

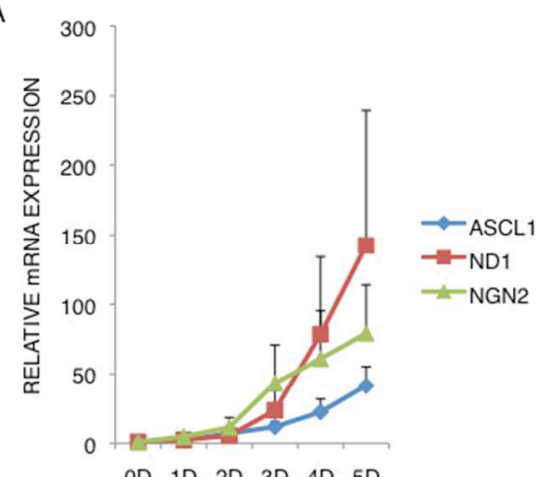

c

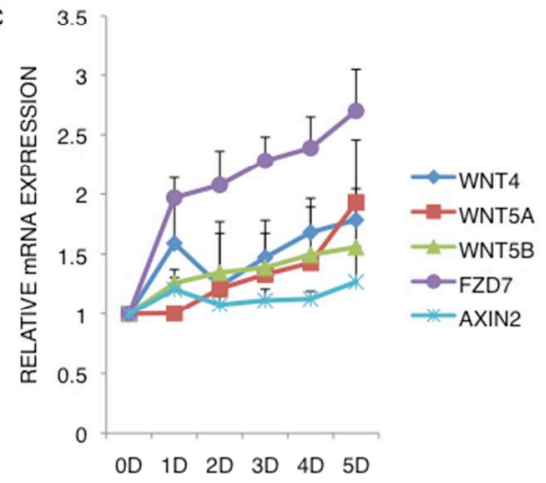

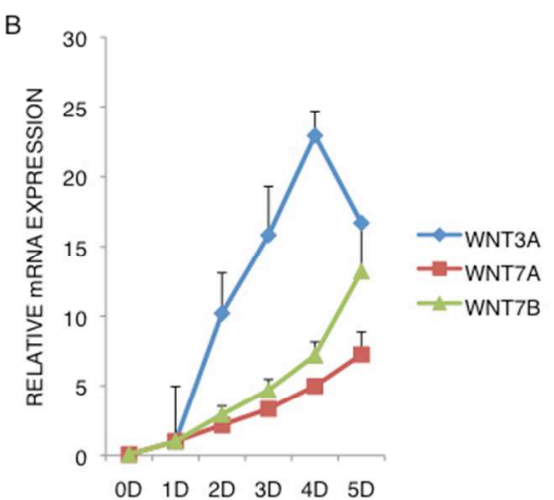

D

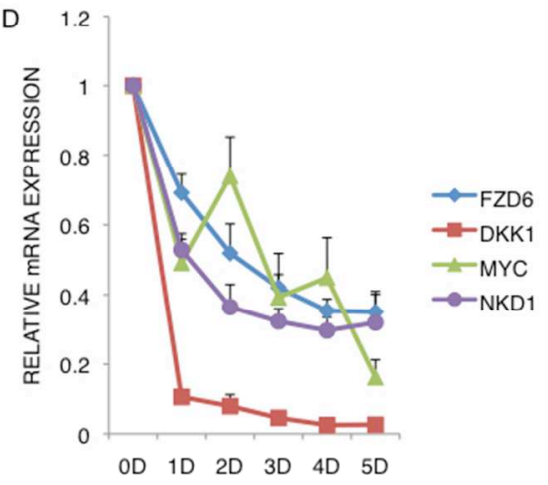

$264 \times 352 \mathrm{~mm}(72 \times 72 \mathrm{DPI})$

ScholarOne Support: (434) 964-4100 
Bengoa, FIG.3
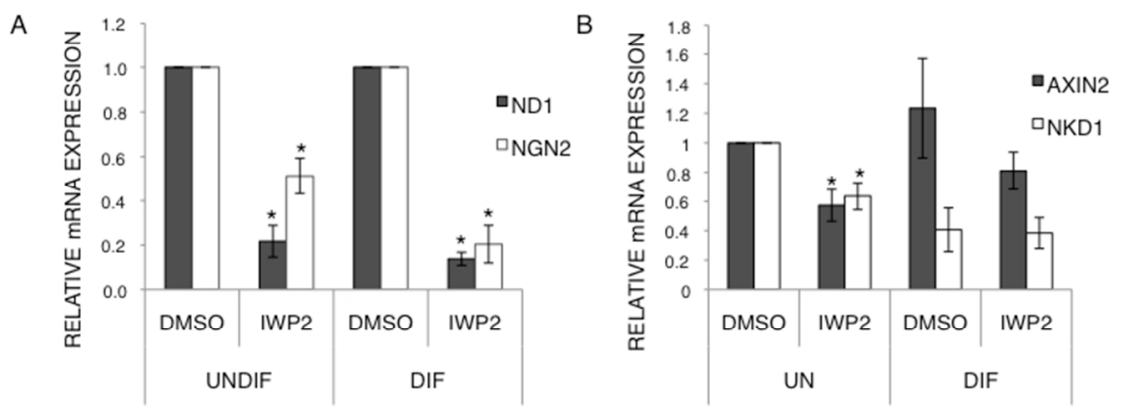

C

D
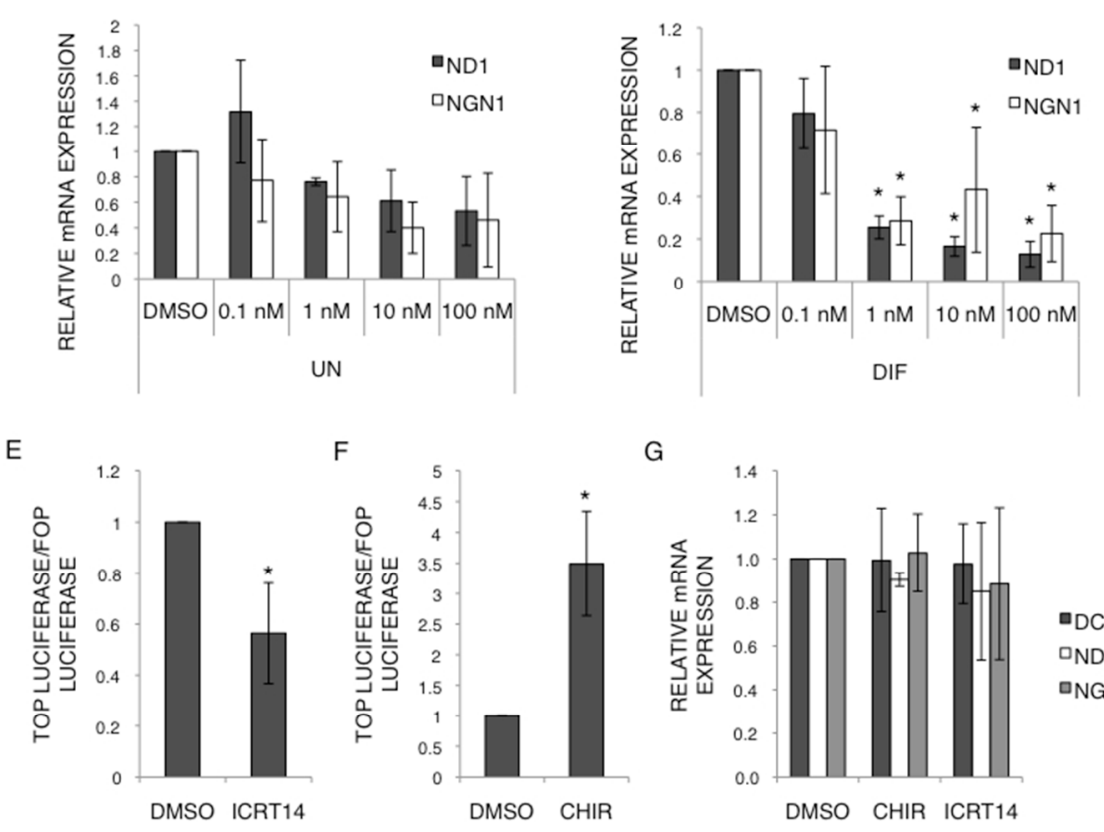

G

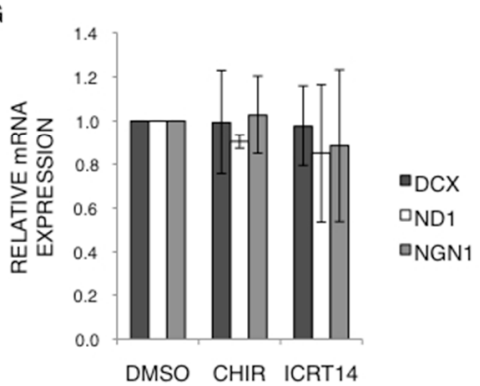

$264 \times 352 \mathrm{~mm}(72 \times 72 \mathrm{DPI})$

ScholarOne Support: (434) 964-4100 


\section{Stem Cells}

Bengoa, FIG.4

A

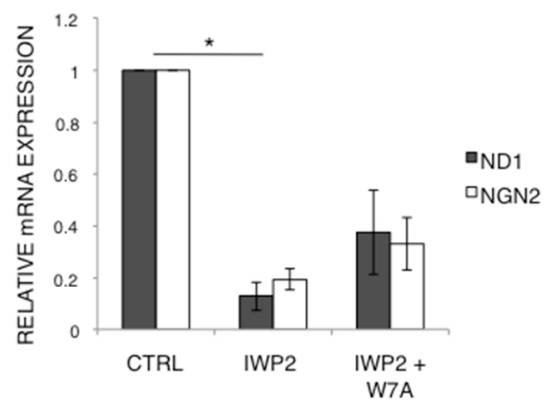

C

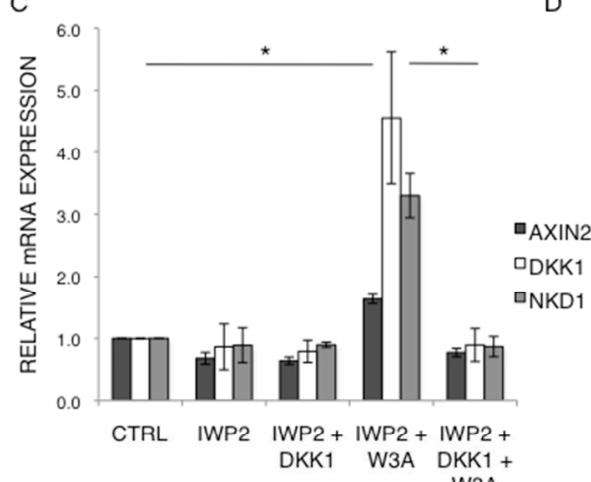

E

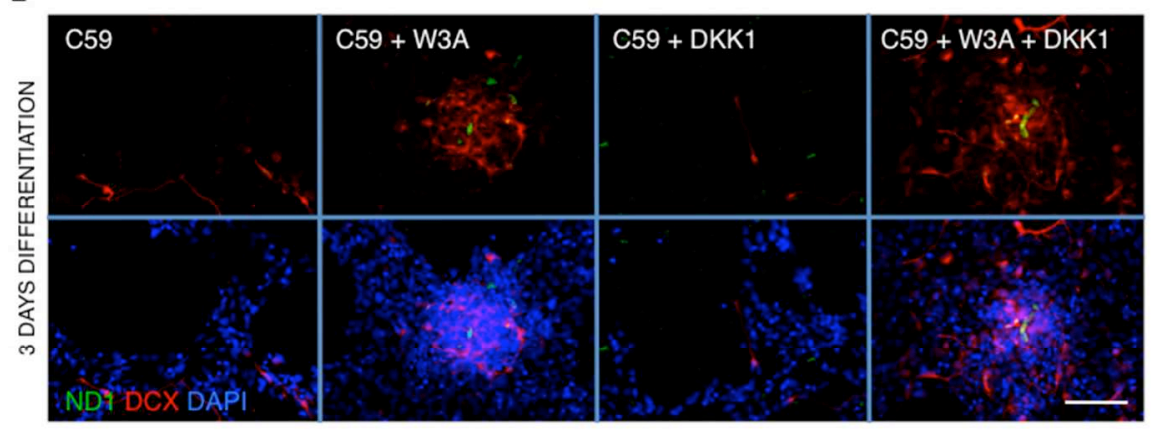

$264 \times 352 \mathrm{~mm}(72 \times 72 \mathrm{DPI})$
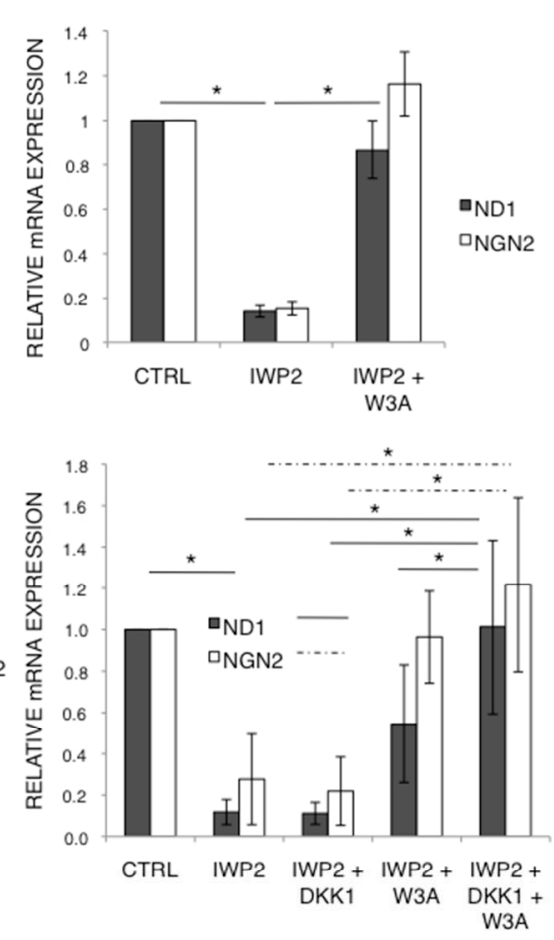

B

DKK1 W3A
WKK 3 A

36

38

39

41

42

43

45

46

47

48

50

33

35

37

10

20

22

24

25
26
27

28

30

$264 \times 352 \mathrm{~mm}(72 \times 72 \mathrm{DPI})$

ScholarOne Support: (434) 964-4100 
Bengoa, FIG.5

A

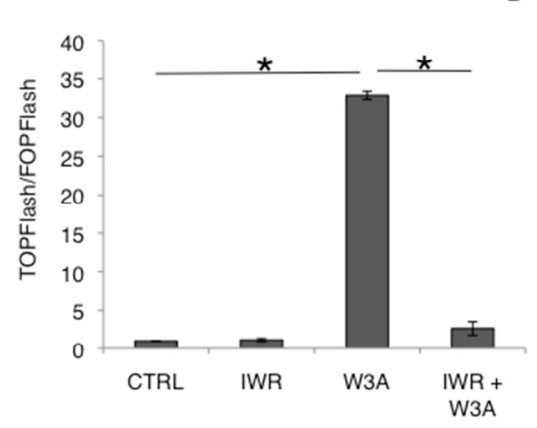

C
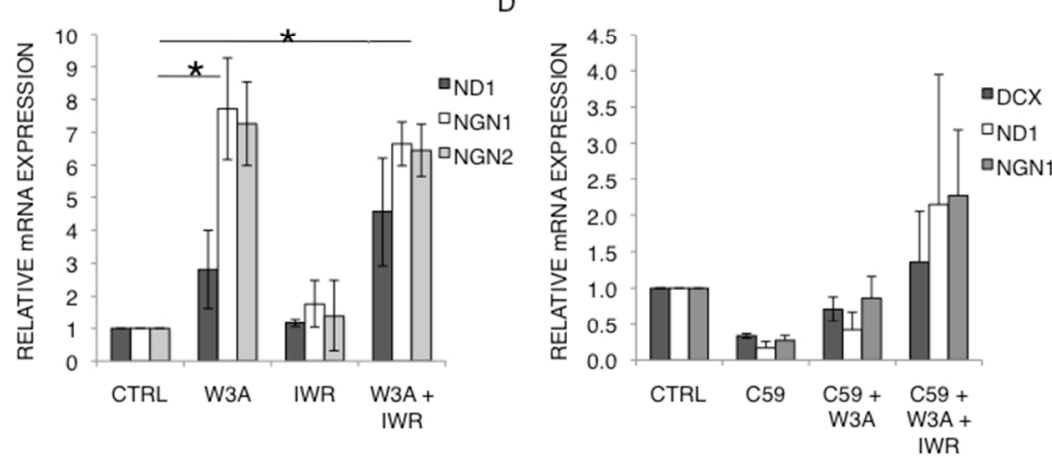

E

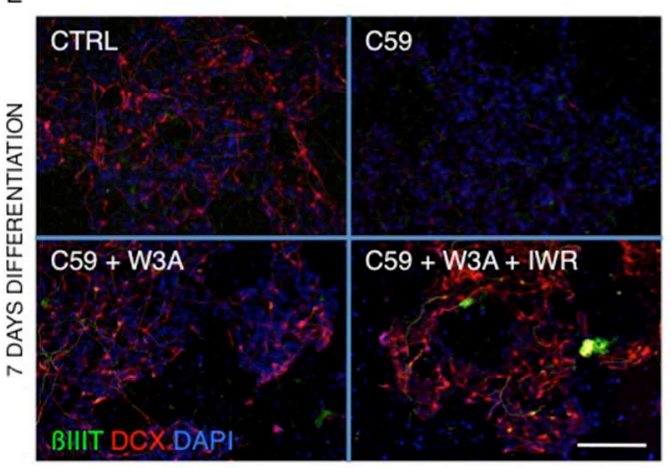

$\mathrm{F}$

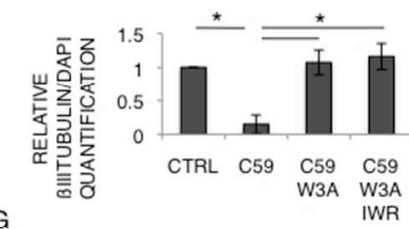

G

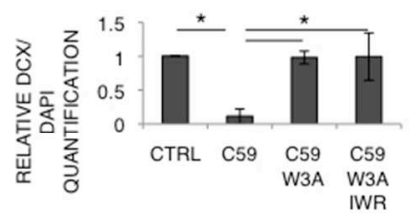

$264 \times 352 \mathrm{~mm}(72 \times 72 \mathrm{DPI})$

ScholarOne Support: (434) 964-4100 


\section{Stem Cells}

A

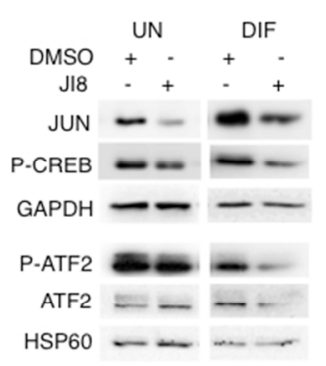

Bengoa, FIG.6

B
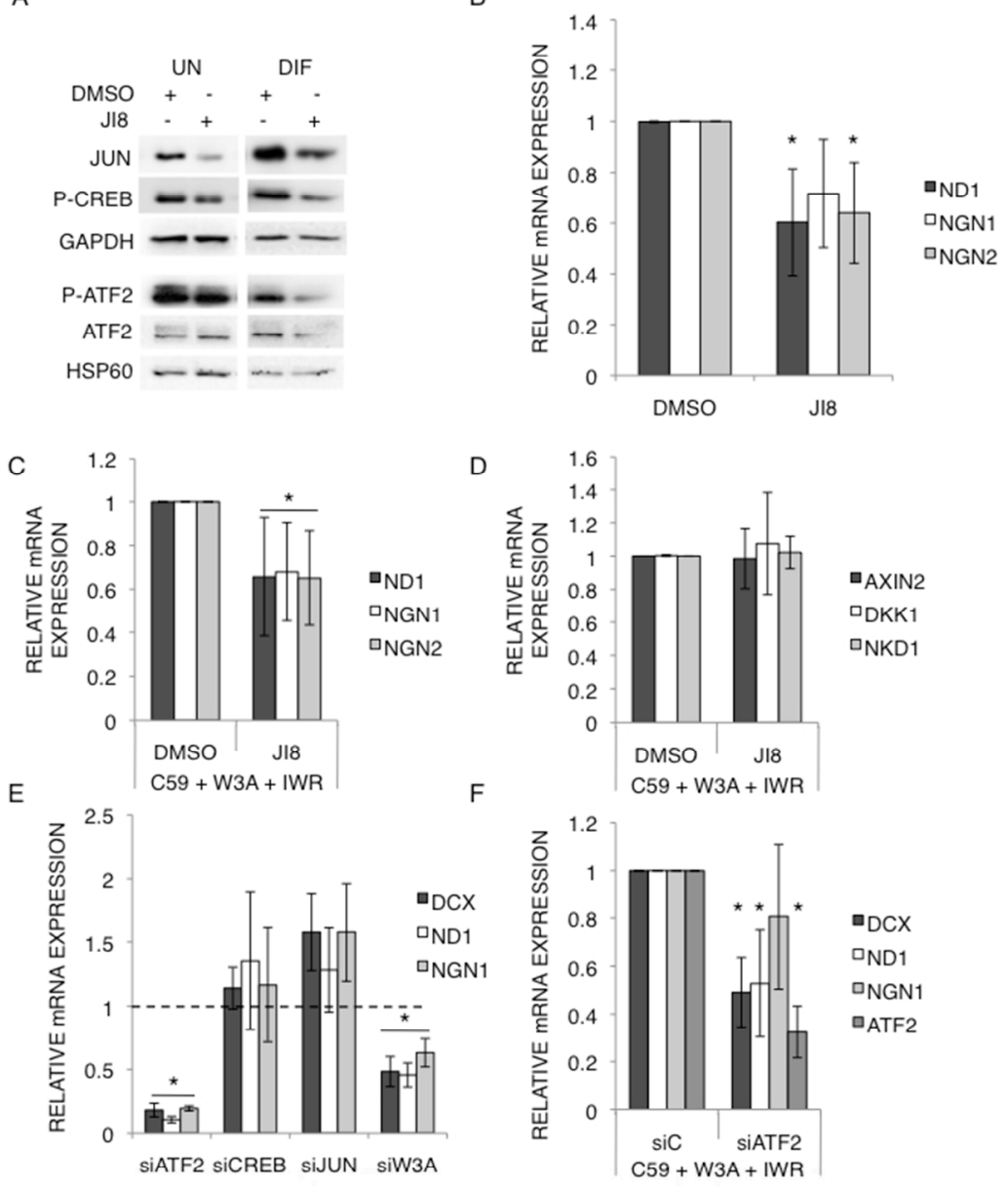

G

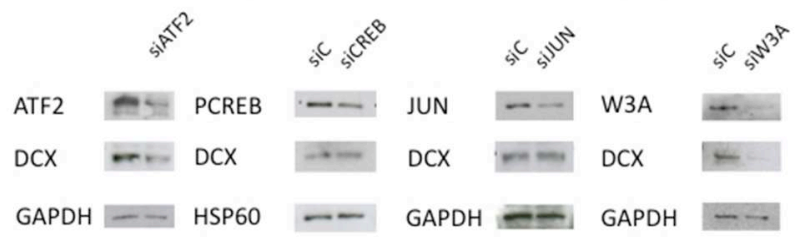

$264 \times 352 \mathrm{~mm}(72 \times 72 \mathrm{DPI})$

ScholarOne Support: (434) 964-4100 
Bengoa, FIG.7

A

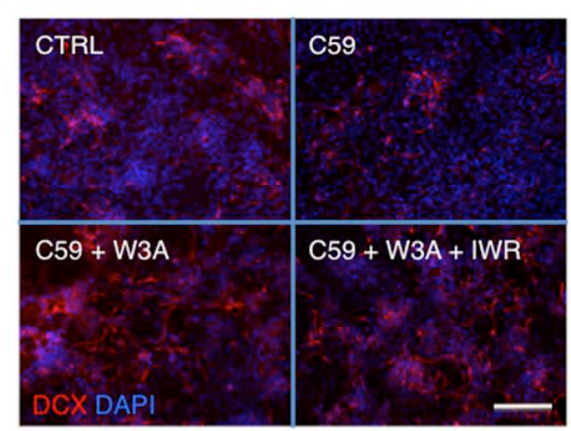

C

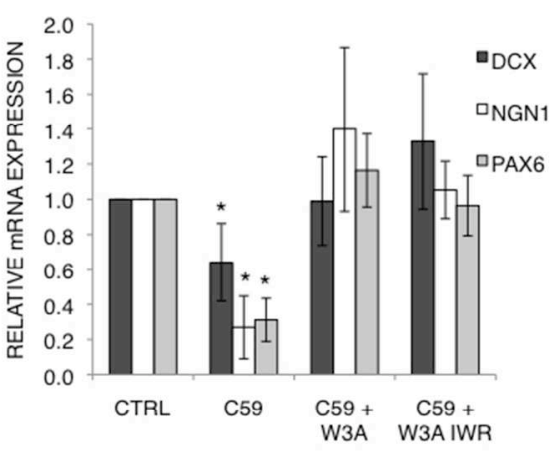

F

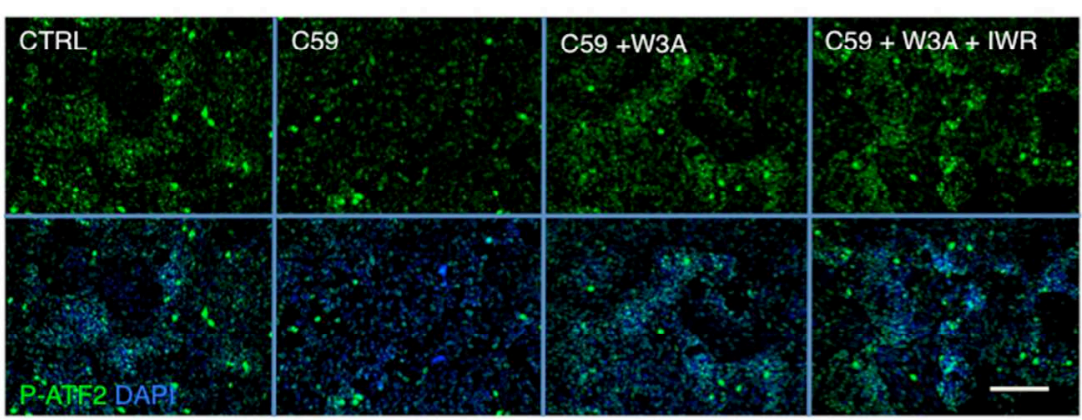

$264 \times 352 \mathrm{~mm}(72 \times 72 \mathrm{DPI})$

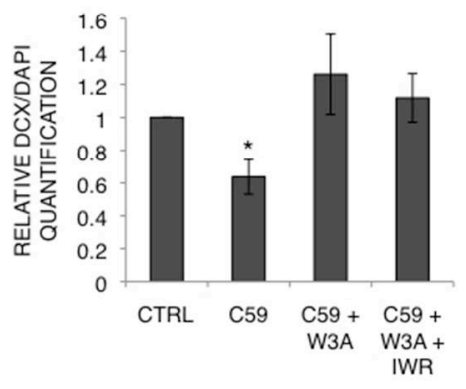

E

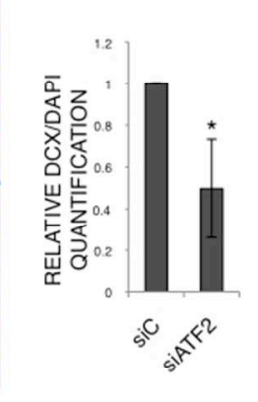

B

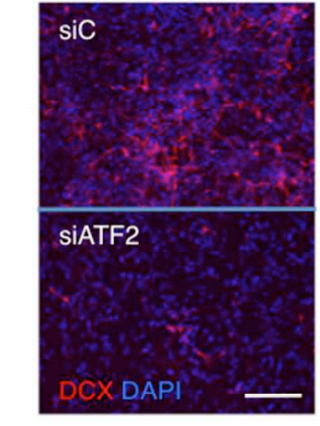

D
33

35

37

38

40

41

43

45

46

47

50

51

52

53

55

56
57
58

59

60 


\section{Supplemental Information}

Supplemental Table 1. Primers used for q-PCR

\begin{tabular}{|c|c|c|}
\hline Primer & Sequence 5 ' $\rightarrow$ 3' & $\begin{array}{l}\text { Primer } \\
\text { Concentration } \\
\text { (nM) }\end{array}$ \\
\hline NEUROGENIN2 F & CGCATCAAGAAGACCCGTAG & 300 \\
\hline NEUROGENIN2 R & GTGAGTGCCCAGATGTAGTTGTG & 300 \\
\hline AXIN2 F & AAGTGCAAACTTTCGCCAAC & 300 \\
\hline AXIN2 R & ACAGGATCGCTCCTCTTGAA & 300 \\
\hline ASCL1 N F & GAGCAGGAGCTTCTCGACTT & 300 \\
\hline ASCL1 N R & CAACGCCACTGACAAGAAAG & 300 \\
\hline NEURO D1 F & ACGAGGAATTCGCCCACGCA & 300 \\
\hline NEURO D1 R & CGCCCATCAGCCCACTCTCG & 300 \\
\hline NEUROGENIN1 F & CGACACCAAGCTCACCAAAA & 900 \\
\hline NEUROGENIN1 R & GCCAGGCGCAGTGTCTC & 900 \\
\hline FZD6 F & TCCCAGATGTATGAAAATGGC & 900 \\
\hline FZD6 R & CCAGATTTGCGAGAGGAAGA & 900 \\
\hline FZD7 F & CGCCTCTGTTCGTCTACCTC & 300 \\
\hline FZD7 R & GTCGTGTTTCATGATGGTGC & 300 \\
\hline WNT7A F & CCGGACTCTCATGAACTTGC & 900 \\
\hline WNT7A R & GAAACTGTGGCAGTGTGGTC & 900 \\
\hline WNT3A F & GTGGAACTGCACCACCGT & 900 \\
\hline WNT3A R & ATGAGCGTGTCACTGCAAAG & 900 \\
\hline DKK1 F & ATGCGTCACGCTATGTGCT & 900 \\
\hline
\end{tabular}


Running title: Wnt signaling switch during neural differentiation

\begin{tabular}{|c|c|c|}
\hline DKK1 R & TCTGGAATACCCATCCAAGG & 900 \\
\hline WNT7B F & TGGCGTCCTGTACGTGAAGCTC & 900 \\
\hline WNT7B R & CGGGGCTAGGCCAGGAATCTT & 900 \\
\hline 36B4 F & GTGTTCGACAATGGCAGCAT & 300 \\
\hline 36B4 R & AGACACTGGCAACATTGCGGA & 300 \\
\hline WNT5B F & GGCTCAGCTTCTGACAGACGCCA & 900 \\
\hline WNT5B R & ACATCTCGGGTCTCTGCACCGG & 900 \\
\hline WNT5A F & GCTCGCTCGGGTGGCGACTTC & 900 \\
\hline WNT5A R & CTTCCAGCCATCCCCAAAGCAACT & 900 \\
\hline MYC F & CACCGAGTCGTAGTCGAGGT & 900 \\
\hline MYC R & TTTCGGGTAGTGGAAAACCA & 900 \\
\hline NKD1 F & ACTTCCAGCCGAAAGTCGT & 900 \\
\hline NKD1 R & CACCATAGGCCGAAGCAC & 900 \\
\hline WNT4 F & AAGGCCATCCTGACACACATG & 300 \\
\hline WNT4 R & GCTAGGCTCCAAGTACACCAGG & 300 \\
\hline DCX F & AAGGACCTGTACCTGCCTCT & 300 \\
\hline DCX R & TGAGCACTCTCCCCTCCTTT & 300 \\
\hline PAX6 F & AGCCCCATATTCGAGCCCCGT & 300 \\
\hline PAX6 R & TGGCCGCCCGTTGACAAAGA & 300 \\
\hline $\mathrm{CD} 44 \mathrm{~F}$ & CAAGTTTTGGTGGCACGCAG & 300 \\
\hline CD44 R & GTGGAATACACCTGCAAAGCG & 300 \\
\hline S100B F & GGGAGACAAGCACAAGCTGAA & 300 \\
\hline S100B R & CATTCGCCGTCTCCATCATTG & 300 \\
\hline OLIG1 F & TACTATGCGGTTTCCCAGGC & 300 \\
\hline
\end{tabular}




\begin{tabular}{|l|l|l|}
\hline OLIG1 R & ACCAGCTCGTAGAGGGAGG & 300 \\
\hline OLIG2 F & CCCCTAAAGGTGCGGATGC & 300 \\
\hline OLIG2 R & CTTTCGCAGGGTCCTCCTTC & 300 \\
\hline PDGFRA F & GGAGGAGAAGTTTCCCAGAGC & 300 \\
\hline PDGFRA R & AGGGTAATGAAAGCTGGCAGA & 300 \\
\hline
\end{tabular}


Running title: Wnt signaling switch during neural differentiation

Supplemental Table 2. Antibodies used for immunofluorescence

\begin{tabular}{|l|l|l|l|}
\hline Antibody & Reference & Species & Dilution \\
\hline NESTIN & Santa Cruz sc-23927 & Mouse & $1: 200$ \\
\hline NANOG & R\&D AF1997 & Goat & $1: 15$ \\
\hline SOX2 & Santa Cruz sc-17320 & Goat & $1: 25$ \\
\hline NEUROD1 & Santa Cruz sc-46684 & Mouse & $1: 50$ \\
\hline ATF2 & Millipore MAB1637 & Mouse & $1: 50$ \\
\hline P-ATF2 & Santa Cruz sc-6233 & Rabbit & $1: 50$ \\
\hline P-ATF2 & Cell Signaling \#5112 & Rabbit & $1: 50$ \\
\hline cJUN & Santa Cruz sc-7982-R & Rabbit & $1: 200$ \\
\hline B-CATENIN & Cell Signaling $\# 9165$ & Rabbit & $1: 25$ \\
\hline P-CREB & Cell Signaling \#9198 & Rabbit & $1: 50$ \\
\hline DCX & Santa Cruz sc-8066 & Goat & $1: 100$ \\
\hline PAX6 & Santa Cruz sc-81649 & Mouse & $1: 100$ \\
\hline
\end{tabular}


Supplemental Table 3. Antibodies used for western blotting

\begin{tabular}{|l|l|l|l|}
\hline Antibody & Reference & Species & Dilution \\
\hline PAX6 & Santa Cruz sc-81649 & Mouse & $1: 1000$ \\
\hline NEUROD1 & Santa Cruz sc-46684 & Mouse & $1: 1000$ \\
\hline BIIITUBULIN & Millipore MAB1637 & Mouse & $1: 2000$ \\
\hline HSP60 & Santa Cruz sc-13966 & Rabbit & $1: 2000$ \\
\hline GAPDH & Sigma G8795 & Mouse & $1: 2000$ \\
\hline cJUN & Cell Signaling $\# 9165$ & Rabbit & $1: 1000$ \\
\hline ATF2 & Santa Cruz sc-6233 & Rabbit & $1: 1000$ \\
\hline P-ATF2 & Santa Cruz sc-7982-R & Rabbit & $1: 1000$ \\
\hline P-CREB & Cell Signaling $\# 9198$ & Rabbit & $1: 1000$ \\
\hline BCAT & BD610154 & Mouse & $1: 2000$ \\
\hline LAM A & Genscript A01455 & Rabbit & $1: 1000$ \\
\hline
\end{tabular}




\section{Legends for supplemental online videos}

Video 1. Wnt7b mRNA expression in the mouse brain ISH data from the Allen Mouse Brain Atlas [50] were visualized using Brain Explorer ${ }^{\circledR}$ software (http://mouse.brainmap.org/static/brainexplorer). The top left corner legend indicates which areas of the mouse brain are being shown, the top right inset is a guide for the actual position of the mouse brain and the bottom right legend is an indicator of the intensity and density of the staining.

Video 2. ATF2 mRNA expression in the mouse brain ISH data from the Allen Mouse Brain Atlas [50] were visualized using Brain Explorer® software (http://mouse.brainmap.org/static/brainexplorer). The top left corner legend indicates which areas of the mouse brain are being shown, the top right inset is a guide for the actual position of the mouse brain and the bottom right legend is an indicator of the intensity and density of the staining. 


\section{Stem Cells}

Running title: Wnt signaling switch during neural differentiation

\section{Supplemental Figure Legends}

\section{Fig. S1. Characterization of hNP cells}

(A-E) Immunofluorescence staining of hNP cells to detect NESTIN (NES, green (A), SOX2 (green) (B), NANOG (red) \& $\beta$ III-tubulin ( $\beta$ IIIT, green) (C), NEUROD1 (ND1, green) (D) and $\beta$-catenin ( $\beta C A T$, red) (E) in undifferentiated (UNDIF) cells and after 3 days of differentiation (DIF); DAPI staining of nuclei is in blue; scale bar $100 \mathrm{um}$. (F) Western blots detecting NEUROD1 (ND1), PAX6 and $\beta$ III-tubulin ( $\beta$ IIIT) in total extracts of hNP cells during 5 days of differentiation; HSP60 was used as a loading control.

\section{Fig. S2. Analysis of changes in Wnt pathway gene expression during hNP cell differentiation using $\operatorname{Taqman}{ }^{\circledR}$ arrays}

(A) mRNA expression of the neuronal genes NEUROD1 (ND1) and ASCL1 in RNA from undifferentiated (UNDIF) or 3 day-differentiated (DIF) hNP cells, as used for analysis in the Taqman ${ }^{\circledR}$ array. (B) Top hits, as defined by fold-change in signal using the Taqman ${ }^{\circledR}$ Human WNT Pathway array, with genes downregulated in differentiating cells in green and upregulated genes in red. (C-D) Graphs show average mRNA expression determined by qPCR of the genes indicated in undifferentiated (UN) or differentiating (DIF) cells and cells treated with DMSO or Wnt-C59 $100 \mathrm{nM}$. (E) mRNA expression determined by q-PCR of the genes indicated in hNP cells treated with vehicle (DMSO and/or CHAPS), Wnt-5a (100 $\mathrm{ng} / \mathrm{ml}$ ) and/or IWP-2 for $72 \mathrm{~h} ;{ }^{*} \mathrm{p}<0,05$ by ANOVA test (error bars represent SD).

\section{Fig. S3. Dkk-1 blocks Wnt/ $\beta$-catenin signaling.}

(A) Cartoon illustrating Dkk-1 inhibition of canonical but not noncanonical Wnt signaling.

(B) Gene reporter assays measuring $\beta$-catenin/Tcf activity (TOPFlash/FOPFlash ratio) in hNP 
cells treated with vehicle (CHAPS), Wnt-3a (100 ng/ml) and/or Dkk-1 (100 ng/ml) for $24 \mathrm{~h}$; * $\mathrm{p}<0,05$ by ANOVA (error bars represent SD).

\section{Fig. S4. Noncanonical Wnt-3a increases phosphorylation and expression of AP-1 family members.}

(A-D) Immunofluorescence staining for phospho-CREB (A), phospho-ATF2 (B), ATF2 (C) and c-Jun (D) in differentiating hNP cells treated Wnt-C59 (100 nM) and vehicle (CHAPS), recombinant Wnt-3a (W3A, $100 \mathrm{ng} / \mathrm{ml})$ and recombinant Dkk-1 (100 ng/ml) or both together; DAPI staining of nuclei is in blue; scale bars $200 \mathrm{um}$.

Fig. S5. Wnt-3a regulation of AP-1 is unaffected by Dkk-1 and reduced by the JNK inhibitor JI8.

(A) Gene reporter assays measuring AP-1 activity in hNP cells treated with vehicle (DMSO) or JI8 (10 uM). (B) Quantification of the 3 independent WB experiments analyzed with ImageJ. (C) Graphs show average mRNA expression determined by q-PCR of the genes indicated, relative to each control siRNA sample; $* \mathrm{p}<0.05$ by $t$ test (error bars represent $\mathrm{SD})$.

Fig. S6. Characterization of iPS-derived neural progenitor cells

(A-C) Immunofluorescence staining of iPS-derived neural progenitor cells to detect DCX (A), NESTIN (green) and SOX2 (red) (B), and PAX6 (green) and P-ATF2 (red) (C) at 5 days of differentiation; DAPI staining of nuclei is in blue; scale bar 200 um. (D) Western blots detecting ND1, PAX6 and DCX in total extracts of iPS-derived neural progenitor cells during 5 days of differentiation; GAPDH was used as a loading control. 
Fig. S7. Neuronal and Wnt pathway gene expression during neuronal differentiation of iPS-derived neural progenitor cells

(A-C) Graphs show average mRNA expression determined by q-PCR of the genes indicated, from 3 independent 7-day time-course experiments (error bars represent SEM). (D) q-PCR of the genes indicated, relative to $36 B 4 ;{ }^{*} \mathrm{p}<0.05$ by $t$ test (error bars represent SD). (E) mRNA expression determined by q-PCR of the genes indicated, relative to $36 B 4$, from 3 experiments using differentiating cells treated for 5 days with vehicle (DMSO and CHAPS), Wnt-C59 $(100 \mathrm{nM})$ and vehicle (CHAPS), Wnt-C59 (100 nM) and recombinant Wnt-3a (100 ng/ml), and Wnt-C59 (100 nM), recombinant Wnt-3a (100 ng/ml) and IWR $(10 \mathrm{uM}) ;{ }^{*} \mathrm{p}<0.05$ by ANOVA test (error bars represent SD).

Fig. S8. Expression of non-canonical Wnt pathway components in mouse dentate gyrus In situ hybridization of $W n t 7 B(\mathrm{~A}), \operatorname{Creb}(\mathrm{B})$ and $A t f 2$ (C) mRNAs in the dentate gyrus from from the Allen Mouse Brain Atlas (http://mouse.brain-map.org [50]).

Fig. S9. Inhibition of alternative pathways during neuronal differentiation of hNP cells (A-B) Graphs show average mRNA expression determined by q-PCR of the genes indicated, upon silencing of the indicated genes; $* \mathrm{p}<0.05$ by ANOVA test (error bars represent SD). (C-D) mRNA expression of neuronal genes in differentiating hNP cells treated with Rho kinase inhibitor Y-27632 and the CAMKII inhibitor myr-AIP, $\mathrm{n}=1$. 
Bengoa, FIG. S1

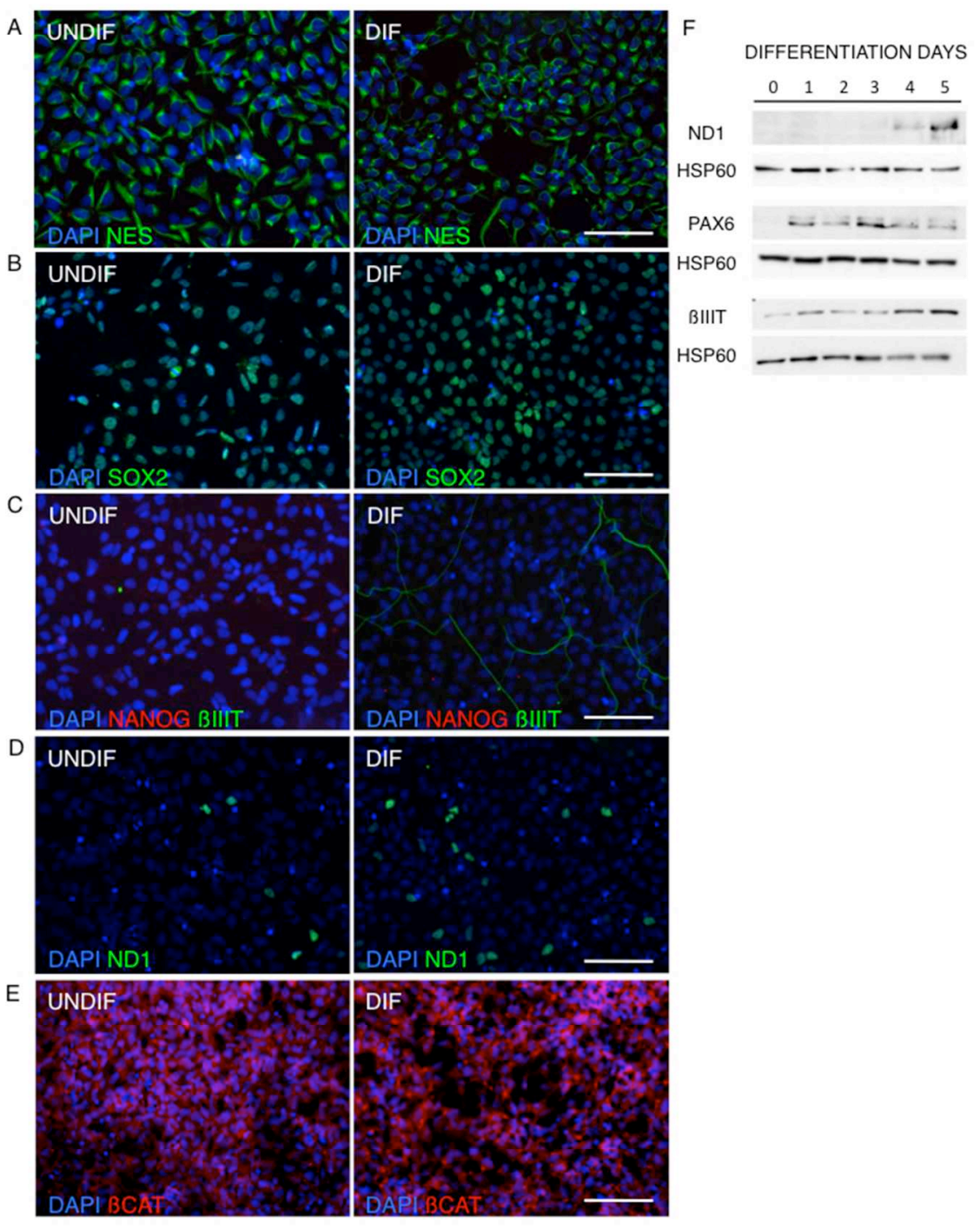

$264 \times 352 \mathrm{~mm}(72 \times 72 \mathrm{DPI})$

ScholarOne Support: (434) 964-4100 


\section{Stem Cells}

A

Bengoa, FIG. S2

$A$

B

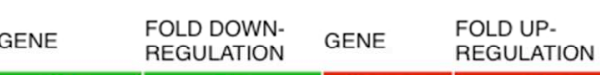

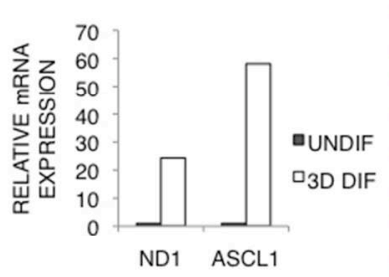

\begin{tabular}{|c|c|c|c|}
\hline WIF1 & 20,4 & WNT3A & 120,5 \\
\hline DKK1 & 12,9 & WNT7A & 17,8 \\
\hline DKK2 & 11,5 & WNT4 & 6,8 \\
\hline TLE2 & 7,8 & SFRP4 & 5,9 \\
\hline FRPB & 5,9 & WNT7B & 4,3 \\
\hline WNT8B & 3,1 & SFRP2 & 3,0 \\
\hline WNT2B & 2,8 & AXIN2 & 2,9 \\
\hline TLE6 & 2,8 & WNT1 & 2,8 \\
\hline CSNK2A1 & 2,7 & WNT5B & 2,7 \\
\hline FZD6 & 2,3 & FZD7 & 2,3 \\
\hline MYC & 2,2 & WNT5A & 1,5 \\
\hline NKD1 & 1,8 & DKK3 & 1,5 \\
\hline
\end{tabular}

C

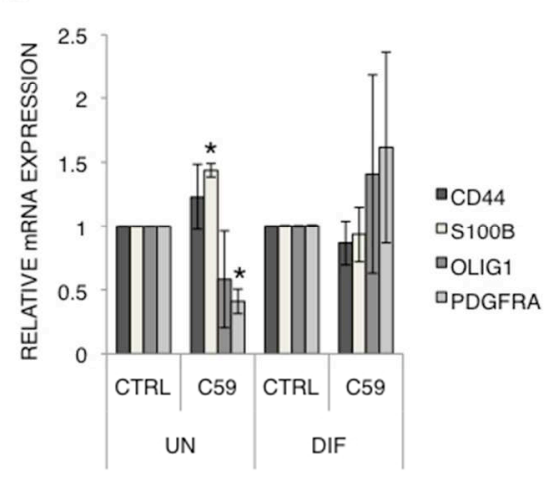

E
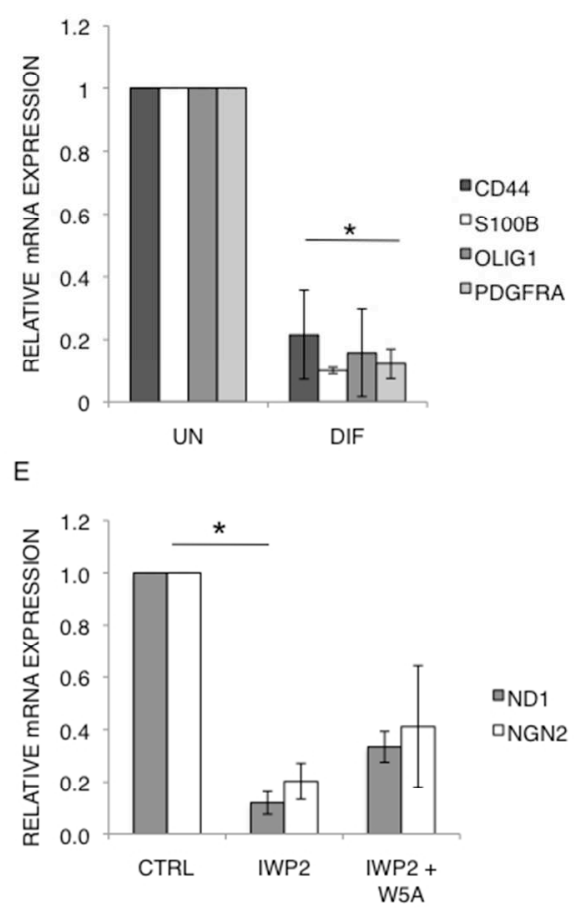

$264 \times 352 \mathrm{~mm}(72 \times 72 \mathrm{DPI})$

ScholarOne Support: (434) 964-4100 
Bengoa, FIG. S3

A

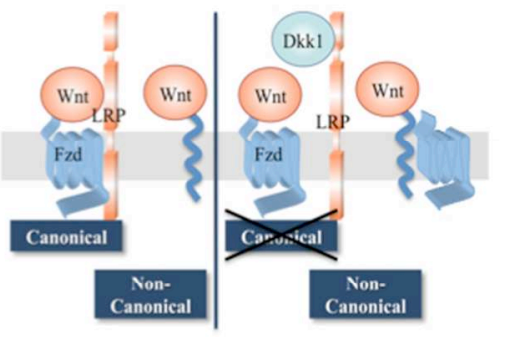

B

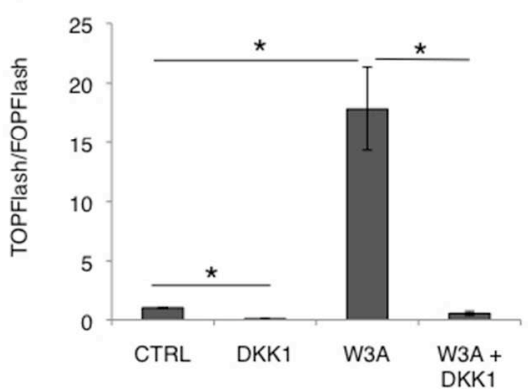

$264 \times 352 \mathrm{~mm}(72 \times 72 \mathrm{DPI})$

ScholarOne Support: (434) 964-4100 


\section{Stem Cells}

Bengoa, FIG. S4

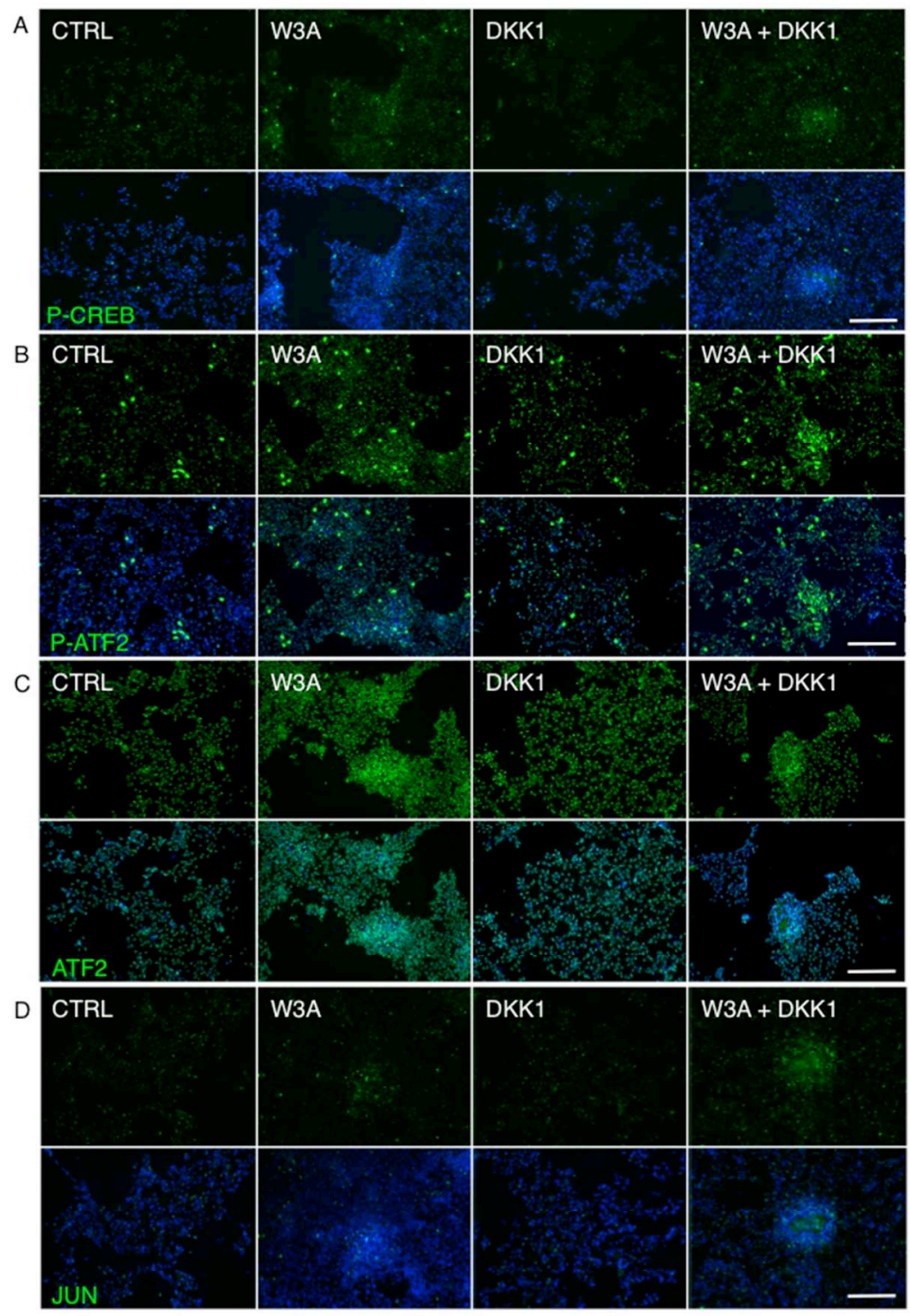

$264 \times 352 \mathrm{~mm}(72 \times 72 \mathrm{DPI})$

ScholarOne Support: (434) 964-4100 
Bengoa, FIG. S5

A

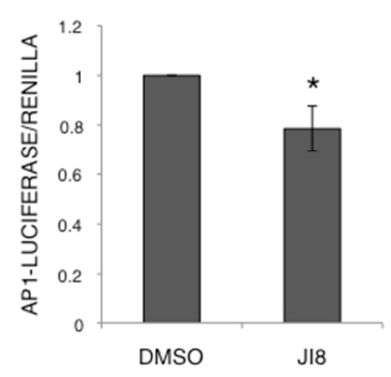

C

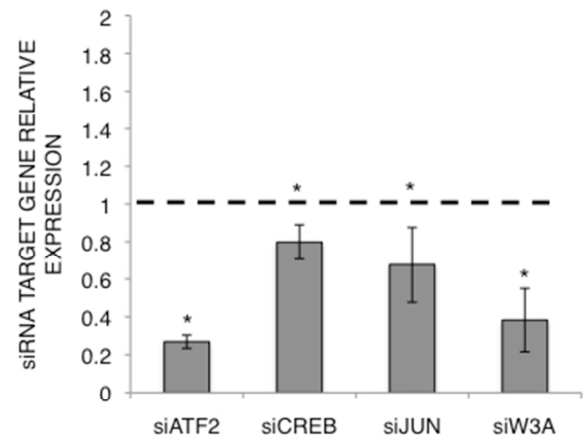

B

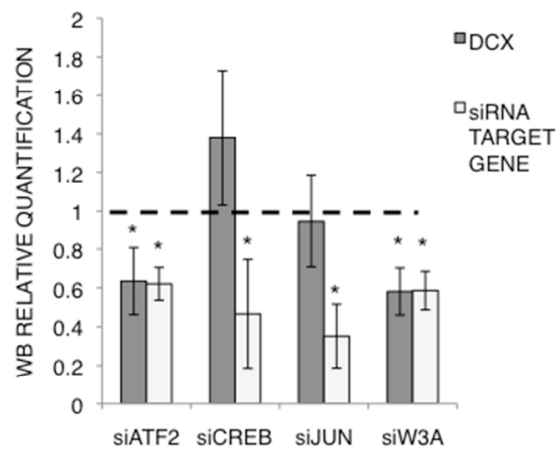

$264 \times 352 \mathrm{~mm}(72 \times 72 \mathrm{DPI})$

ScholarOne Support: (434) 964-4100 


\section{Stem Cells}

Bengoa, FIG. S6
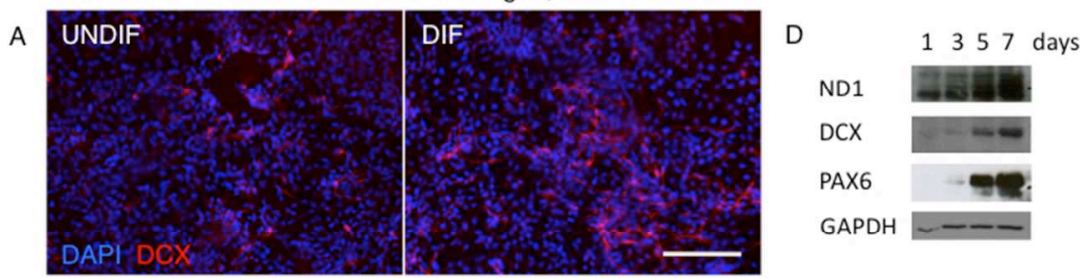

B

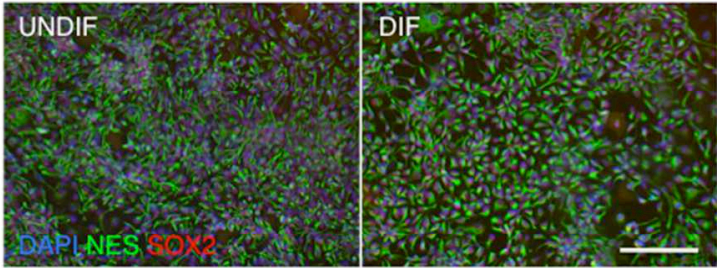

C

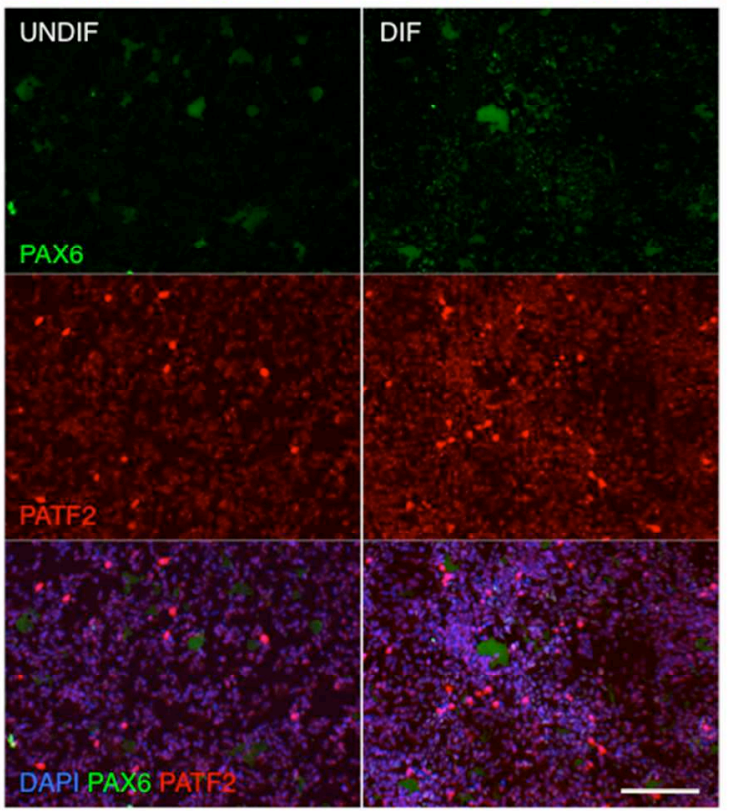

$264 \times 352 \mathrm{~mm}(72 \times 72 \mathrm{DPI})$

ScholarOne Support: (434) 964-4100 
Bengoa, FIG. S7

A
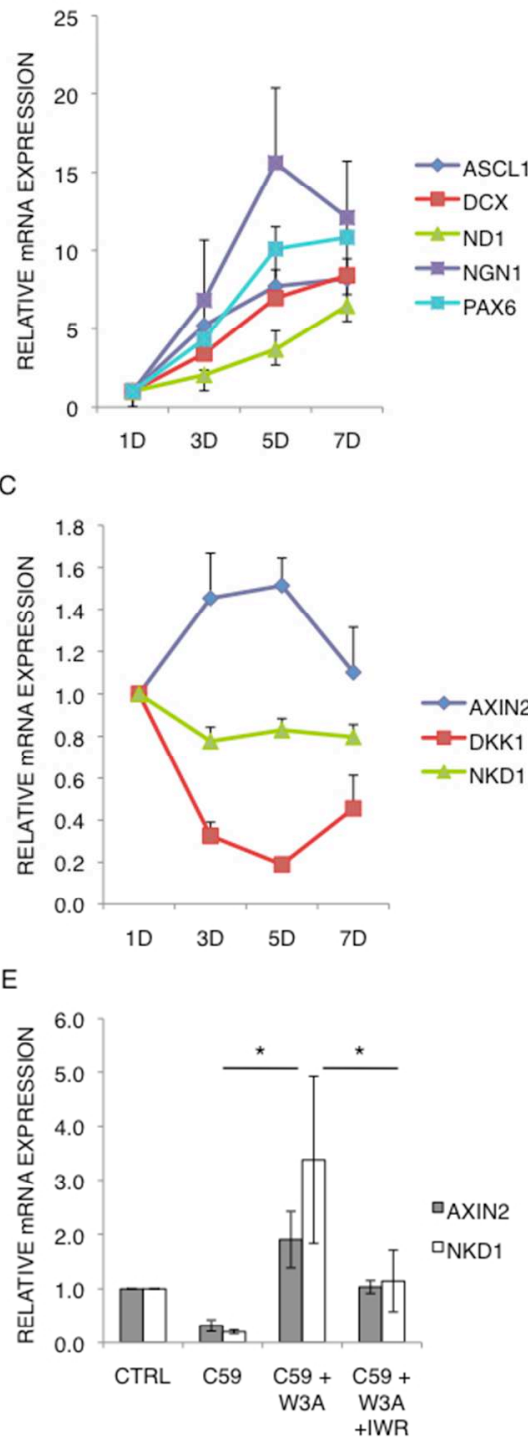

B

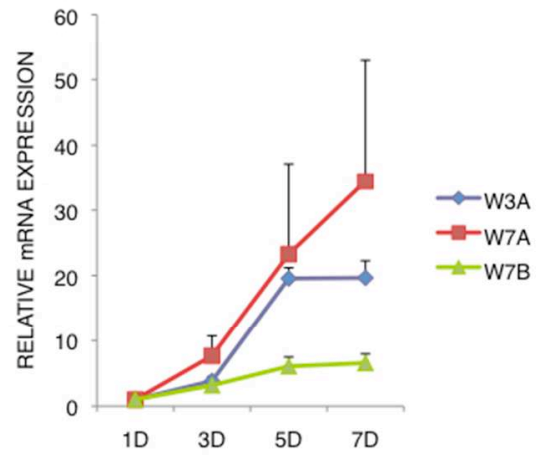

D

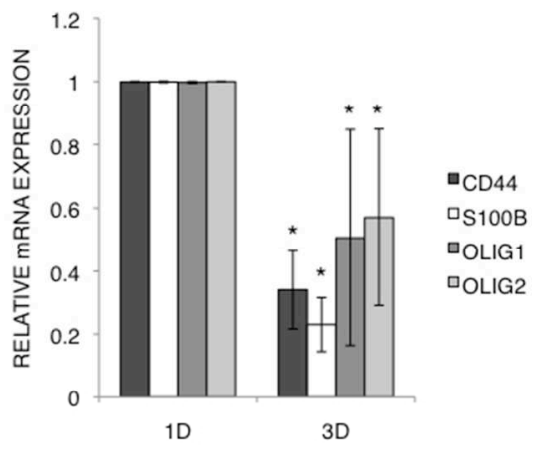

$264 \times 352 \mathrm{~mm}(72 \times 72 \mathrm{DPI})$

ScholarOne Support: (434) 964-4100 


\section{Stem Cells}

Bengoa, FIG. S8

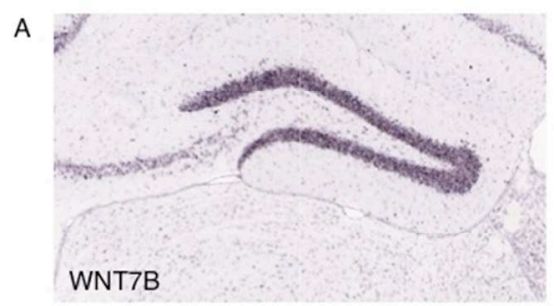

B

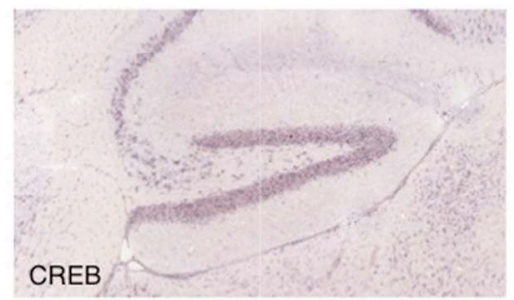

C

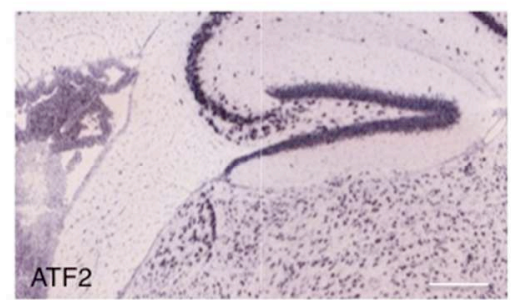

$264 \times 352 \mathrm{~mm}(72 \times 72$ DPI $)$

ScholarOne Support: (434) 964-4100 
Bengoa, FIG. S9
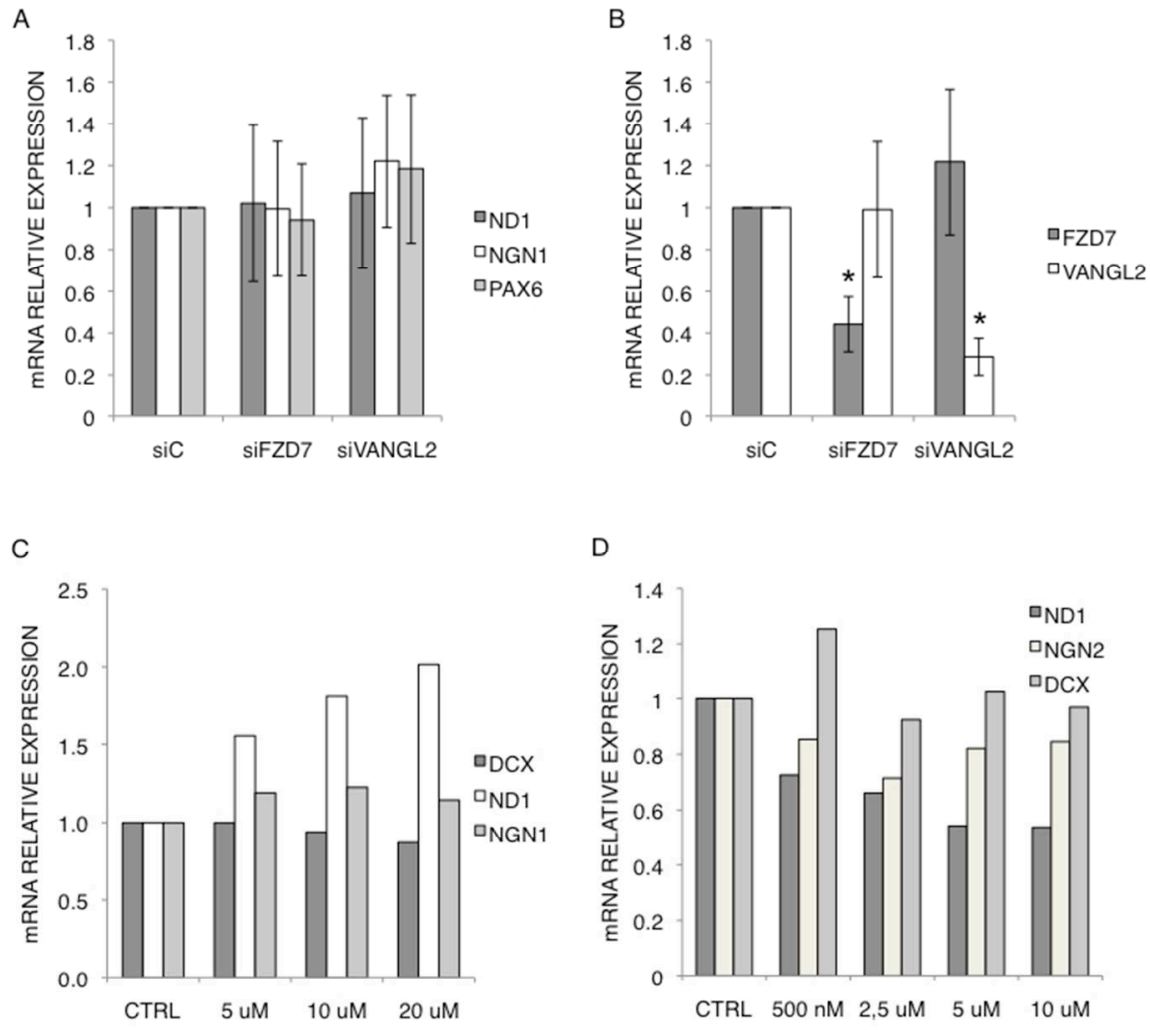

$264 \times 352 \mathrm{~mm}(72 \times 72 \mathrm{DPI})$

ScholarOne Support: (434) 964-4100 